JOURNAL OF

FUNCTION SPACES AND APPLICATIONS

Volume 8, Number 1 (2010), 17-54
(C) 2010, Scientific Horizon http://www.jfsa.net

\title{
Asymptotic analysis for a weakly damped wave equation with application to a problem arising in elasticity
}

\section{Gabriel Nguetseng, Hubert Nnang and Nils Svanstedt}

(Communicated by Lars-Erik Persson)

2000 Mathematics Subject Classification. 35B40.

Keywords and phrases. Homogenization, two-scale convergence, elasticity, wave equation.

Abstract. The present work is devoted to the study of homogenization of the weakly damped wave equation

$$
\begin{gathered}
\int_{\Omega} \rho \frac{\partial^{2} u_{\varepsilon}}{\partial t^{2}}(t) \cdot v d x+2 \varepsilon^{2} \mu \int_{\Omega_{f}^{\varepsilon}} E_{i j}\left(\frac{\partial u_{\varepsilon}}{\partial t}(t)\right) E_{i j}(v) d x \\
+\varepsilon^{2} \lambda \int_{\Omega_{f}^{\varepsilon}} \operatorname{div}\left(\frac{\partial u_{\varepsilon}}{\partial t}(t)\right) \operatorname{div} v d x+\vartheta \int_{\Omega_{f}^{\varepsilon}} \operatorname{div}\left(u_{\varepsilon}(t)\right) \operatorname{div} v d x \\
=\int_{\Omega} f(t) \cdot v d x \text { for all } v=\left(v^{1}, v^{2}, v^{3}\right) \in V_{\varepsilon}(0<t<T),
\end{gathered}
$$

with initial conditions

$$
u_{\varepsilon}(0)=\frac{\partial u_{\varepsilon}}{\partial t}(0)=\omega\left(\text { the origin in } \mathbb{R}^{3}\right)
$$

Convergence homogenization results are achieved using the two-scale convergence theory. 


\section{Introduction}

Our main objective in the present work is to prove a homogenization theorem for the weakly damped wave equation

$$
\begin{aligned}
& \int_{\Omega} \rho^{\varepsilon} \frac{\partial^{2} u_{\varepsilon}}{\partial t^{2}}(t) \cdot v d x+\varepsilon^{2} b^{\varepsilon}\left(\frac{\partial u_{\varepsilon}}{\partial t}(t), v\right)+a^{\varepsilon}\left(u_{\varepsilon}(t), v\right)=\int_{\Omega} f(t) \cdot v d x \\
& \text { for all } v=\left(v^{1}, v^{2}, v^{3}\right) \in V_{\varepsilon}(0<t<T)
\end{aligned}
$$

with initial conditions

$$
u_{\varepsilon}(0)=\frac{\partial u_{\varepsilon}}{\partial t}(0)=\omega\left(\text { the origin in } \mathbb{R}^{3}\right),
$$

where $\Omega$ is a smooth bounded open set in the numerical space $\mathbb{R}_{x}^{3}$ of variables $x=\left(x_{1}, x_{2}, x_{3}\right), T$ is a fixed positive real, the dot denotes the usual Euclidean inner product in $\mathbb{R}^{3}, f=\left(f^{1}, f^{2}, f^{3}\right)$ is given in $L^{2}\left(0, T ; \mathbb{L}^{2}(\Omega ; \mathbb{R})\right)$ (with $\left.\mathbb{L}^{2}(\Omega ; \mathbb{R})=L^{2}(\Omega ; \mathbb{R})^{3}\right), d x$ denotes the Lebesgue measure on $\mathbb{R}_{x}^{3}$, the functions $\rho^{\varepsilon}, a^{\varepsilon}, b^{\varepsilon}$, and the set $\Omega_{s}^{\varepsilon}$ are defined farther on and

(1.3) $V_{\varepsilon}=\left\{v \in \mathbb{H}_{0}^{1}(\Omega ; \mathbb{R})=H_{0}^{1}(\Omega ; \mathbb{R})^{3}: E_{i j}(v)=0\right.$ in $\left.\Omega_{s}^{\varepsilon}(i, j=1,2,3)\right\}$

with $E_{i j}(v)=\frac{1}{2}\left(\frac{\partial v^{i}}{\partial x_{j}}+\frac{\partial v^{j}}{\partial x_{i}}\right) \quad i, j=1,2,3$.

In what follows, all vector spaces $F$ are assumed to be complex vector spaces, and $\mathbb{F}$ denotes the product $F \times F \times F$ (three times). If $X$ denotes a locally compact space, we will write $\mathcal{C}(X)=\mathcal{C}(X ; \mathbb{C})$ for the space of continuous mappings from $X$ into $\mathbb{C}$ (complex numbers) and $\mathcal{B}(X)=\mathcal{B}(X ; \mathbb{C})$ for that of bounded continuous mappings from $X$ into $\mathbb{C}$.

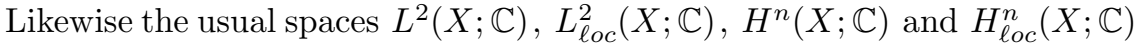
(integer $n \geq 1, X$ provided with a positive Radon measure $\beta$ ) will be denoted by $L^{2}(X), L_{\ell o c}^{2}(X), H^{n}(X)$ and $H_{\ell o c}^{n}(X)$. The corresponding subspaces of real functions will be denoted by $L^{2}(X ; \mathbb{R}), L_{\ell o c}^{2}(X ; \mathbb{R})$, $H^{n}(X ; \mathbb{R})$ and $H_{\ell o c}^{n}(X ; \mathbb{R})$. In the definitions of other function spaces, the functions are assumed to be measurable. If $A$ is any Banach space, $\mathcal{C}(X ; A)$ will denote the space of all continuous functions from $X$ into $A$, and $L^{2}(X ; A)$ will denote the space of all functions $u: X \rightarrow A$ with $\int_{X}\|u(x)\|_{A}^{2} d \beta(x)<\infty,\|\cdot\|_{A}$ being the norm on $A$. Furthermore, we systematically use the convention of Einstein. For basic concepts and notations concerning integration theory we refer to $[3,4]$. 
Let us now give a precise description of the problem. Let $\alpha, \lambda, \mu, \rho_{s}$, $\rho_{f}, \vartheta, c_{0}, b_{i j k h}$ be real numbers with

$$
b_{i j k h}=\lambda \delta_{i j} \delta_{k h}+\mu\left(\delta_{i k} \delta_{j h}+\delta_{i h} \delta_{j k}\right) \text { for } i, j, k, h=1,2,3
$$

( $\delta_{i j}$ being the Kronecker symbol), and

$$
0<\alpha<1, \mu>0, \rho_{s}>0, \rho_{f}>0, \frac{\lambda}{\mu} \geq\left(-\frac{2}{3}\right) \alpha, \vartheta=c_{0}^{2} \rho_{f}
$$

Then the symmetry condition

$$
b_{i j k h}=b_{j i k h}=b_{i j h k}=b_{k h i j}
$$

and the ellipticity condition

$$
\begin{aligned}
& b_{i j k h} \xi_{k h} \xi_{i j} \geq \delta \xi_{i j} \xi_{i j}(\text { with } \delta=2 \mu(1-\alpha)) \text { for all symmetric } \\
& \text { matrices of real numbers }\left(\xi_{i j}\right)
\end{aligned}
$$

follow immediately. Let $Y_{s}$ be a smooth open set in $\mathbb{R}_{y}^{3}$ (the 3 -dimensional numerical space of variables $\left.y=\left(y_{1}, y_{2}, y_{3}\right)\right)$ such that

(1.8) $\bar{Y}_{s} \subset Y=\left(-\frac{1}{2}, \frac{1}{2}\right) \times\left(-\frac{1}{2}, \frac{1}{2}\right) \times\left(-\frac{1}{2}, \frac{1}{2}\right)\left(\bar{Y}_{s}\right.$ the closure of $\left.Y_{s}\right)$,

and let $Y_{f}$ be the open set $Y \backslash \bar{Y}_{s}$. Let $\rho \in L^{\infty}\left(\mathbb{R}_{y}^{3}\right)$ be given by

$$
\rho(y)=\rho_{s} \text { in } \widetilde{Y}_{s} \text { and } \rho_{f} \text { in } \tilde{Y}_{f},
$$

where, denoting by $\mathbb{Z}$ the set of integers, we denote

$$
\tilde{Y}_{s}=\bigcup_{k \in \mathbb{Z}^{3}}\left(k+Y_{s}\right) \text { and } \tilde{Y}_{f}=\mathbb{R}_{y}^{3} \backslash \overline{\widetilde{Y}}_{s}\left(\overline{\widetilde{Y}}_{s} \text { the closure of } \widetilde{Y}_{s} \text { in } \mathbb{R}_{y}^{3}\right) .
$$

For fixed $\varepsilon>0$, let $\rho^{\varepsilon}$ be the function in $L^{\infty}\left(\mathbb{R}_{x}^{3}\right)$ defined by

$$
\rho^{\varepsilon}(x)=\rho\left(\frac{x}{\varepsilon}\right) \quad\left(x \in \mathbb{R}^{3}\right) .
$$

Let $\Omega$ be as above. We endow $\mathbb{H}^{1}(\Omega ; \mathbb{R})=H^{1}(\Omega ; \mathbb{R})^{3}$ with the norm

$$
\||v|\|=\left(\int_{\Omega} v^{i} v^{i} d x+\int_{\Omega} E_{i j}(v) E_{i j}(v) d x\right)^{\frac{1}{2}} \quad\left(v=\left(v^{i}\right) \in \mathbb{H}^{1}(\Omega ; \mathbb{R})\right) .
$$


Thanks to Korn's inequality (see [14]), the norm $\||\cdot|\|$ is equivalent to the usual $\mathbb{H}^{1}(\Omega)$-norm; and the seminorm

$$
\|\mid v\|_{0}=\left(\int_{\Omega} E_{i j}(v) E_{i j}(v) d x\right)^{\frac{1}{2}} \quad\left(v \in \mathbb{H}^{1}(\Omega ; \mathbb{R})\right)
$$

is a norm in $\mathbb{H}_{0}^{1}(\Omega ; \mathbb{R})$ equivalent to the $\mathbb{H}^{1}(\Omega)$-norm.

For $\varepsilon>0$, we set

$$
\Omega_{s}^{\varepsilon}=\Omega \cap \varepsilon \widetilde{Y}_{s} \text { and } \Omega_{f}^{\varepsilon}=\Omega \cap \varepsilon \tilde{Y}_{f},
$$

which defines two open subsets of $\Omega$. Let $b^{\varepsilon}$ and $a^{\varepsilon}$ be the two continuous bilinear forms on $\mathbb{H}^{1}(\Omega ; \mathbb{R}) \times \mathbb{H}^{1}(\Omega ; \mathbb{R})$ defined by

$b^{\varepsilon}(u, v)=\int_{\Omega_{f}^{\varepsilon}} b_{i j k h} \frac{\partial u^{k}}{\partial x_{h}} \frac{\partial v^{i}}{\partial x_{j}} d x=\lambda \int_{\Omega_{f}^{\varepsilon}} \operatorname{div} u \operatorname{div} v d x+2 \mu \int_{\Omega_{f}^{\varepsilon}} E_{i j}(u) E_{i j}(v) d x$ and

$$
a^{\varepsilon}(u, v)=\vartheta \int_{\Omega_{f}^{\varepsilon}} \operatorname{div} u \operatorname{div} v d x, \text { respectively, }
$$

where $\operatorname{div} u=\frac{\partial u^{1}}{\partial x_{1}}+\frac{\partial u^{2}}{\partial x_{2}}+\frac{\partial u^{3}}{\partial x_{3}}$ for $u=\left(u^{i}\right)$. It is clear that the space $V_{\varepsilon}$ in $(1.3)$ is a closed vector subspace of $\mathbb{H}_{0}^{1}(\Omega ; \mathbb{R})$, and the seminorm

$$
\||v|\|_{\varepsilon}=\left(\int_{\Omega_{f}^{\varepsilon}} E_{i j}(v) E_{i j}(v) d x\right)^{\frac{1}{2}} \quad\left(v \in \mathbb{H}_{0}^{1}(\Omega ; \mathbb{R})\right)
$$

is a norm on $V_{\varepsilon}$ equivalent to $\||\cdot|\|_{0}$.

By the Galerkin method one can prove the following existence and uniqueness result (see [2] or [13]) :

Theorem 1. For each given $\varepsilon>0$, there exists one and only one function $u_{\varepsilon}$ from $[0, T]$ into $V_{\varepsilon}$ defined by (1.1)-(1.2) and satisfying $u_{\varepsilon} \in \mathcal{C}\left([0, T] ; V_{\varepsilon}\right), \quad \frac{\partial u_{\varepsilon}}{\partial t} \in \mathcal{C}\left([0, T] ; \mathbb{L}^{2}(\Omega ; \mathbb{R})\right) \cap L^{2}\left(0, T ; V_{\varepsilon}\right), \quad \frac{\partial^{2} u_{\varepsilon}}{\partial t^{2}} \in$ $L^{2}\left(0, T ; \mathbb{L}^{2}(\Omega ; \mathbb{R})\right)$.

The periodic homogenization of (1.1)-(1.2), together with many other hyperbolic problems, is studied in Sanchez-Palencia [14]. Here, the homogenization method is the pioneering approach using multiple-scale asymptotic expansions. We also refer to [11] for the homogenization, by means of two-scale convergence, of a mixture of solid and fluid. Another study of interest on this topic is presented in [8], where the homogenization of hyperbolic-parabolic equations in perforated domains is worked out. 
The paper is organized as follows. Section 2 is devoted to some fundamentals of homogenization. In section 3 we study the homogenization problem for (1.2)-(1.2) in the periodic setting when $f \in \mathcal{C}^{1}\left([0, T] ; \mathbb{L}^{2}(\Omega ; \mathbb{R})\right)$ with $f(0)=\omega$. Section 4 is concerned with the study of the periodic homogenization problem for (1.1)-(1.2) in the general case $f \in$ $L^{2}\left(0, T ; \mathbb{L}^{2}(\Omega ; \mathbb{R})\right)$.

\section{Fundamentals of homogenization}

The aim is to study the homogenization of (1.1)-(1.2) by using the twoscale convergence method (see $[1,8,12]$ ). We will need the notion of weak two-scale convergence in $L^{2}(Q)$. That is, given $\left(v_{\varepsilon}\right)_{\varepsilon>0} \subset L^{2}(Q)$ and $v_{0} \in L^{2}\left(Q ; L_{\text {per }}^{2}(Z)\right)$, where

$$
\begin{aligned}
& Q=\Omega \times(0, T), \\
& Z=Y \times \Theta \text { with } \Theta=\left(-\frac{1}{2}, \frac{1}{2}\right), \\
& L_{\text {per }}^{2}(Z)=\left\{v \in L_{\text {loc }}^{2}\left(\mathbb{R}^{4}\right): v \text { is } Z \text {-periodic }\right\},
\end{aligned}
$$

we will say that $v_{\varepsilon} \rightarrow v_{0}$ in $L^{2}(Q)$-weak two-scale if, as $\varepsilon \rightarrow 0$,

$$
\int_{Q} v_{\varepsilon} \Phi^{\varepsilon} d x d t \rightarrow \iint_{Q \times Z} v_{0} \Phi d x d t d y d \tau
$$

for every $\Phi \in L^{2}\left(Q ; \mathcal{C}_{\text {per }}(Z)\right), \mathcal{C}_{\text {per }}(Z)=\left\{v \in \mathcal{C}\left(\mathbb{R}^{4}\right): v\right.$ is $Z$-periodic $\}$, where $\Phi^{\varepsilon}(x, t)=\Phi\left(x, t, \frac{x}{\varepsilon}, \frac{t}{\varepsilon}\right)$ in the sense of [12, Proposition 4.1], $(x, t) \in$ $Q, \varepsilon>0$. In particular $(2.1)$ makes sense for $\Phi \in L^{2}\left(Q ; \mathcal{C}_{\text {per }}(Y)\right) \otimes \mathcal{C}_{\text {per }}(\Theta)$, or for $\Phi \in \mathcal{C}\left(\bar{Q} ; L_{p e r}^{\infty}(Z)\right)$ [12, Proposition 4.5].

We recall the following fundamental results :

Lemma 1. Let $E$ be a fundamental sequence, i.e., $E$ is an ordinary sequence of positive real numbers tending to 0 ; and suppose the sequence $\left(v_{\varepsilon}\right)_{\varepsilon \in E} \subset L^{2}(Q)$ is bounded in $L^{2}(Q)$. Then, a subsequence $E^{\prime}$ can be extracted from $E$ such that the sequence $\left(v_{\varepsilon}\right)_{\varepsilon \in E^{\prime}}$ is weakly two-scale convergent in $L^{2}(Q)$.

Proof. See, e.g., [7].

Lemma 2. Let $\left(v_{\varepsilon}\right)_{\varepsilon \in E}$ be a bounded sequence in $L^{2}(Q)$. Suppose that $\left(\varepsilon D v_{\varepsilon}\right)_{\varepsilon \in E}$ is bounded in $\mathbb{L}^{2}(Q)$ (where $\left.D v_{\varepsilon}=\left(\frac{\partial v_{\varepsilon}}{\partial x_{1}}, \frac{\partial v_{\varepsilon}}{\partial x_{2}}, \frac{\partial v_{\varepsilon}}{\partial x_{3}}\right)\right)$. Then, there exist a subsequence $E^{\prime} \subset E$ and a function $v_{0} \in L^{2}\left(Q ; L_{\text {per }}^{2}(Z)\right)$ 
with $\frac{\partial v_{0}}{\partial y_{i}} \in L^{2}\left(Q ; L_{p e r}^{2}(Z)\right)$ such that, as $E^{\prime} \ni \varepsilon \rightarrow 0$,

$$
\int_{Q} v_{\varepsilon} \Phi^{\varepsilon} d x d t \rightarrow \iint_{Q \times Z} v_{0} \Phi d x d t d y d \tau
$$

and

$$
\int_{Q} \varepsilon \frac{\partial v_{\varepsilon}}{\partial x_{i}} \Phi^{\varepsilon} d x d t \rightarrow \iint_{Q \times Z} \frac{\partial v_{0}}{\partial y_{i}} \Phi d x d t d y d \tau, \quad i=1,2,3
$$

for all $\Phi \in L^{2}\left(Q ; \mathcal{C}_{\text {per }}(Z)\right)$.

Proof. See, e.g., $[1,13]$.

Lemma 3. Let $\left(v_{\varepsilon}\right)_{\varepsilon \in E}$ be a bounded sequence in $L^{2}(Q)$. Suppose that $\left(\frac{\partial v_{\varepsilon}}{\partial t}\right)_{\varepsilon \in E}$ is bounded in $L^{2}(Q)$. Then, there exist a subsequence $E^{\prime} \subset E$ and two functions $v_{0} \in H^{1}\left(0, T ; L^{2}\left(\Omega ; L_{p e r}^{2}(Y)\right)\right)$ and $v_{1} \in L^{2}\left(Q ; L_{\text {per }}^{2}\left(Y ; H_{\#}^{1}(\Theta)\right)\right)$ (with $H_{\#}^{1}(\Theta)=\left\{v \in H_{\text {per }}^{1}(\Theta)\right.$ : $\left.\int_{\Theta} v d \tau=0\right\}, H_{\text {per }}^{1}(\Theta)=\left\{v \in H_{\ell o c}^{1}(\mathbb{R}): v, \frac{d v}{d \tau}\right.$ are $\Theta$-periodic $\}$, and $L_{\text {per }}^{2}\left(Y ; H_{\#}^{1}(\Theta)\right)=\left\{v \in L_{\ell o c}^{2}\left(\mathbb{R}^{3} ; H_{\#}^{1}(\Theta)\right): v\right.$ is $Y$-periodic $\left.\}\right)$, such that as $E^{\prime} \ni \varepsilon \rightarrow 0$,

$$
\int_{Q} v_{\varepsilon} \Phi^{\varepsilon} d x d t \rightarrow \iint_{Q \times Z} v_{0} \Phi d x d t d y d \tau
$$

and

$$
\int_{Q} \frac{\partial v_{\varepsilon}}{\partial t} \Phi^{\varepsilon} d x d t \rightarrow \iint_{Q \times Z}\left(\frac{\partial v_{0}}{\partial t}+\frac{\partial v_{1}}{\partial \tau}\right) \Phi d x d t d y d \tau
$$

for all $\Phi \in L^{2}\left(Q ; \mathcal{C}_{\text {per }}(Z)\right)$.

Proof. Based on Lemma 1, there exist a subsequence $E^{\prime} \subset E$ and two functions $v_{0}, w_{0} \in L^{2}\left(Q ; L_{p e r}^{2}(Z)\right)$ such that, as $E^{\prime} \ni \varepsilon \rightarrow 0$,

$$
\begin{aligned}
& \int_{Q} v_{\varepsilon} \Phi^{\varepsilon} d x d t \rightarrow \iint_{Q \times Z} v_{0} \Phi d x d t d y d \tau \text { and } \\
& \int_{Q} \frac{\partial v_{\varepsilon}}{\partial t} \Phi^{\varepsilon} d x d t \rightarrow \iint_{Q \times Z} w_{0} \Phi d x d t d y d \tau
\end{aligned}
$$

for every $\Phi \in L^{2}\left(Q ; \mathcal{C}_{p e r}(Z)\right)$.

Let us prove (2.2). This will be do if we show that $v_{0}$ in (2.4) does not depend to $\tau \in \mathbb{R}$. For $\varphi \in \mathcal{D}(Q), \psi \in \mathcal{C}_{\text {per }}(Y)$ and $\theta \in \mathcal{C}_{\text {per }}^{\infty}(\Theta)$ arbitrarily fixed, we have

$$
\int_{Q} \frac{\partial v_{\varepsilon}}{\partial t} \varphi \psi^{\varepsilon} \theta^{\varepsilon} d x d t=-\int_{Q} v_{\varepsilon} \frac{\partial \varphi}{\partial t} \psi^{\varepsilon} \theta^{\varepsilon} d x d t-\frac{1}{\varepsilon} \int_{Q} v_{\varepsilon} \varphi \psi^{\varepsilon}\left(\frac{d \theta}{d \tau}\right)^{\varepsilon} d x d t .
$$


Taking into account the two limits in (2.4), it follows that

$$
\int_{Q} v_{\varepsilon} \varphi \psi^{\varepsilon}\left(\frac{d \theta}{d \tau}\right)^{\varepsilon} d x d t \rightarrow 0 \text { as } E^{\prime} \ni \varepsilon \rightarrow 0
$$

that is,

$$
\iint_{Q \times Z} v_{0} \varphi \otimes \psi \otimes \frac{d \theta}{d \tau} d x d t d y d \tau=0
$$

Then, for almost all $(x, t, y) \in Q \times Y$,

$$
\int_{\Theta} v_{0}(x, t, y, \cdot) \frac{d \theta}{d \tau} d \tau=0 \text { for all } \theta \in \mathcal{C}_{p e r}^{\infty}(\Theta) .
$$

Therefore $\frac{\partial v_{0}(x, t, y, \cdot)}{\partial \tau}=0$ (a.e. in $\left.Q \times Y\right)$. Hence $v_{0}$ does not depend to $\tau$. In other words, $v_{0} \in L^{2}\left(Q ; L_{p e r}^{2}(Y)\right)$ and we deduce (2.2).

Let us now prove (2.3). By taking in (2.4) $\Phi=\varphi \otimes \psi \otimes 1, \varphi \in \mathcal{D}(Q)$ and $\psi \in \mathcal{C}_{\text {per }}(Y)$, it follows that

$$
\int_{Q} v_{\varepsilon}(x, t) \varphi(x, t) \psi\left(\frac{x}{\varepsilon}\right) d x d t \rightarrow \iint_{Q \times Y} v_{0}(x, t, y) \varphi(x, t) \psi(y) d x d t d y
$$

and

$$
\int_{Q} \frac{\partial v_{\varepsilon}}{\partial t}(x, t) \varphi(x, t) \psi\left(\frac{x}{\varepsilon}\right) d x d t \rightarrow \iint_{Q \times Z} w_{0}(x, t, y, \tau) \varphi(x, t) \psi(y) d x d t d y d \tau .
$$

But we have

$$
\int_{Q} \frac{\partial v_{\varepsilon}}{\partial t}(x, t) \varphi(x, t) \psi\left(\frac{x}{\varepsilon}\right) d x d t=-\int_{Q} v_{\varepsilon}(x, t) \frac{\partial \varphi}{\partial t}(x, t) \psi\left(\frac{x}{\varepsilon}\right) d x d t
$$

so that, letting $E^{\prime} \ni \varepsilon \rightarrow 0$, the above limits drive us to

$$
\iint_{Q \times Z} w_{0}(x, t, y, \tau) \varphi(x, t) \psi(y) d x d t d y d \tau=-\iint_{Q \times Y} v_{0}(x, t, y) \frac{\partial \varphi}{\partial t}(x, t) \psi(y) d x d t d y .
$$

Thus, we have $\frac{\partial v_{0}}{\partial t} \in L^{2}\left(Q ; L_{\text {per }}^{2}(Y)\right)$ with

$$
\int_{\Theta}\left(w_{0}(x, t, y, \tau)-\frac{\partial v_{0}}{\partial t}(x, t, y)\right) d \tau=0
$$

for almost all $(x, t, y) \in Q \times Y$. Thus, there exists a function $u_{1}$ from $Q \times Y$ into $H_{\#}^{1}(\Theta)=\left\{v \in H_{\text {per }}^{1}(\Theta): \int_{\Theta} v d \tau=0\right\} \quad\left(\right.$ with $H_{\text {per }}^{1}(\Theta)=\{v \in$ 
$H_{\ell o c}^{1}(\mathbb{R}): v, \frac{d v}{d \tau}$ are $\Theta$-periodic $\left.\}\right)$ such that

$$
\left\{\begin{array}{l}
-\frac{d^{2} u_{1}(x, t, y, \cdot)}{d \tau^{2}}=w_{0}(x, t, y, \cdot)-\frac{\partial v_{0}}{\partial t}(x, t, y) \text { in } \Theta=\left(-\frac{1}{2}, \frac{1}{2}\right), \\
\frac{d u_{1}\left(x, t, y,-\frac{1}{2}\right)}{d \tau}=\frac{d u_{1}\left(x, t, y, \frac{1}{2}\right)}{d \tau}=0
\end{array}\right.
$$

a.e. in $Q \times Y$. In fact, the above boundary value problem is an homogeneous Neumann problem, and the semi-norm $w \rightarrow\left(\int_{\Theta}\left|\frac{d w}{d \tau}\right|^{2}\right)^{\frac{1}{2}}$ is equivalent to the $H^{1}(\Theta)$-norm in $H_{\#}^{1}(\Theta)$; so that we can apply Lax-Milgram Lemma. By letting $-\frac{d u_{1}(x, t, y, \cdot)}{d \tau}=v_{1}(x, t, y, \cdot)$, it follows from $(2.5)$ that $w_{0}(x, t, y, \cdot)=$ $\frac{\partial v_{0}}{\partial t}(x, t, y)+\frac{\partial v_{1}}{\partial \tau}(x, t, y, \cdot)$ a.e. $Q \times Y$; and (2.3) follows.

By successive iterations, we can generalize the above lemma as follows

Lemma 4. Let $\left(v_{\varepsilon}\right)_{\varepsilon \in E}$ be a bounded sequence in $L^{2}(Q)$. Suppose that for some integer $n \geq 1$, $\left(\frac{\partial^{j} v_{\varepsilon}}{\partial t^{j}}\right)_{\varepsilon \in E}$ is bounded in $L^{2}(Q), j=1, \cdots, n$. Then, there exist a subsequence $E^{\prime} \subset E$ and two functions $v_{0} \in H^{n}\left(0, T ; L^{2}(\Omega\right.$; $\left.\left.L_{\text {per }}^{2}(Y)\right)\right)$ and $v_{1} \in L^{2}\left(Q ; L_{\text {per }}^{2}\left(Y ; H_{\#}^{n}(\Theta)\right)\right)$ with $H_{\#}^{n}(\Theta)=\{u \in$ $\left.H_{\text {per }}^{n}(\Theta): \int_{\Theta} u d \tau=0\right\}$, such that as $E^{\prime} \ni \varepsilon \rightarrow 0$,

$$
\int_{Q} \frac{\partial^{j} v_{\varepsilon}}{\partial t^{j}} \Phi^{\varepsilon} d x d t \rightarrow \iint_{Q \times Z} \frac{\partial^{j} v_{0}}{\partial t^{j}} \Phi d x d t d y d \tau, \quad j=0, \cdots, n-1
$$

and

$$
\int_{Q} \frac{\partial^{n} v_{\varepsilon}}{\partial t^{n}} \Phi^{\varepsilon} d x d t \rightarrow \iint_{Q \times Z}\left(\frac{\partial^{n} v_{0}}{\partial t^{n}}+\frac{\partial^{n} v_{1}}{\partial \tau^{n}}\right) \Phi d x d t d y d \tau
$$

for all $\Phi \in L^{2}\left(Q ; \mathcal{C}_{\text {per }}(Z)\right)$.

\section{The homogenization result in the case}

$$
f \in \mathcal{C}^{1}\left([0, T] ; \mathbb{L}^{2}(\Omega ; \mathbb{R})\right), f(0)=\omega
$$

The preliminary results below are based on the following general estimates, which will be easily proved by using a Gronwall lemma.

Lemma 5. Let $\left(u_{\varepsilon}\right)_{\varepsilon>0}$ be the sequence of functions defined in Theorem 1. Then,

$$
\begin{aligned}
& \sup _{\varepsilon>0}\left(\left\|u_{\varepsilon}^{\prime}\right\|_{L^{\infty}\left(0, T ; \mathbb{L}^{2}(\Omega)\right)}+\left\|\varepsilon u_{\varepsilon}^{\prime}\right\|_{L^{2}\left(0, T ; \mathbb{H}_{0}^{1}(\Omega)\right)}+\left\|\operatorname{div} u_{\varepsilon}\right\|_{L^{\infty}\left(0, T ; L^{2}(\Omega)\right)}\right)<+\infty \\
& \text { and } \\
& \sup _{\varepsilon>0}\left(\left\|u_{\varepsilon}\right\|_{L^{\infty}\left(0, T ; \mathbb{L}^{2}(\Omega)\right)}+\left\|\varepsilon u_{\varepsilon}\right\|_{L^{\infty}\left(0, T ; \mathbb{H}_{0}^{1}(\Omega)\right)}\right)<+\infty .
\end{aligned}
$$


Furthermore, if $f \in \mathcal{C}^{1}\left([0, T] ; \mathbb{L}^{2}(\Omega)\right)$ with $f(0)=\omega$, then

$\sup _{\varepsilon>0}\left(\left\|u_{\varepsilon}^{\prime \prime}\right\|_{L^{\infty}\left(0, T ; \mathbb{L}^{2}(\Omega)\right)}+\left\|\varepsilon u_{\varepsilon}^{\prime \prime}\right\|_{L^{2}\left(0, T ; \mathbb{H}_{0}^{1}(\Omega)\right)}+\left\|\operatorname{div} u_{\varepsilon}^{\prime}\right\|_{L^{\infty}\left(0, T ; L^{2}(\Omega)\right)}\right)<+\infty$.

Remark 1. The condition $f \in \mathcal{C}^{1}\left([0, T] ; \mathbb{L}^{2}(\Omega ; \mathbb{R})\right)$ with $f(0)=\omega$ allows us to prove that the solution $u_{\varepsilon}$ of (1.2)-(1.2) is also a solution of the problem (see [13])

$$
\begin{aligned}
& \int_{\Omega} \rho^{\varepsilon} \frac{\partial^{3} u_{\varepsilon}^{i}}{\partial t^{3}}(t) v^{i} d x+\varepsilon^{2} b^{\varepsilon}\left(\frac{\partial^{2} u_{\varepsilon}}{\partial t^{2}}(t), v\right)+a^{\varepsilon}\left(\frac{\partial u_{\varepsilon}}{\partial t}(t), v\right)=\int_{\Omega} \frac{\partial f^{i}}{\partial t}(t) v^{i} d x \\
& \text { for all } v=\left(v^{i}\right) \in V_{\varepsilon} \quad(0<t<T) \\
& \qquad u_{\varepsilon}(0)=\frac{\partial u_{\varepsilon}}{\partial t}(0)=\frac{\partial^{2} u_{\varepsilon}}{\partial t^{2}}(0)=\omega .
\end{aligned}
$$

Then, the last estimate becomes obvious. Further, the choice of $f$ as an element of $\mathcal{C}^{1}\left([0, T] ; \mathbb{L}^{2}(\Omega ; \mathbb{R})\right)$ with $f(0)=\omega$, will permits us to study the weakly two-scale convergence of the sequence of second derivative $\left(u_{\varepsilon}^{\prime \prime}\right)_{\varepsilon>0}$.

3.1 Preliminaries. In the sequel $E$ denotes a fundamental sequence (see Lemma 1).

Lemma 6. There exist a sequence $E^{\prime} \subset E$ and two functions $p_{0} \in$ $L^{2}\left(Q ; L_{p e r}^{2}(Y)\right)$ with $\frac{\partial p_{0}}{\partial t} \in L^{2}\left(Q ; L_{p e r}^{2}(Y)\right)$, and $u_{0}=\left(u_{0}^{1}, u_{0}^{2}, u_{0}^{3}\right) \in$ $L^{2}\left(Q ; \mathbb{H}_{\text {per }}^{1}(Y)\right)$ with $\frac{\partial u_{0}}{\partial t} \in L^{2}\left(Q ; \mathbb{H}_{\text {per }}^{1}(Y)\right), \frac{\partial^{2} u_{0}}{\partial t^{2}} \in L^{2}\left(Q ; \mathbb{L}_{\text {per }}^{2}(Y)\right)$, such that, as $E^{\prime} \ni \varepsilon \rightarrow 0$,

$$
\begin{aligned}
& u_{\varepsilon}^{i} \rightarrow u_{0}^{i} \text { in } L^{2}(Q) \text {-weak two-scale, } \\
& p_{\varepsilon} \rightarrow p_{0} \text { in } L^{2}(Q) \text {-weak two-scale, }
\end{aligned}
$$

where $p_{\varepsilon}=-\vartheta \operatorname{div} u_{\varepsilon}$. Furthermore, we have

$$
\begin{gathered}
e_{i j}\left(u_{0}\right)=e_{i j}\left(\frac{\partial u_{0}}{\partial t}\right)=p_{0}=0 \text { in } Q \times Y_{s}, \\
\operatorname{div}_{y} u_{0}=\operatorname{div}_{y}\left(\frac{\partial u_{0}}{\partial t}\right)=0 \text { in } Q \times Y_{f} \\
\int_{Y_{f}} p_{0} d y+\vartheta \operatorname{div}\left(\int_{Y} u_{0} d y\right)=0 \text { in } Q .
\end{gathered}
$$


where $e_{i j}\left(u_{0}\right)=\frac{1}{2}\left(\frac{\partial u_{0}^{i}}{\partial y_{j}}+\frac{\partial u_{0}^{j}}{\partial y_{i}}\right), i, j=1,2,3, \operatorname{div}_{y} u_{0}=e_{i i}\left(u_{0}\right)=\frac{\partial u_{0}^{1}}{\partial y_{1}}+$ $\frac{\partial u_{0}^{2}}{\partial y_{2}}+\frac{\partial u_{0}^{3}}{\partial y_{3}}$ and $H_{p e r}^{1}(Y)=\left\{v \in H_{\ell o c}^{1}\left(\mathbb{R}^{3}\right): v, \frac{\partial v}{\partial y_{i}}(i=1,2,3)\right.$ are $Y$ periodic\}.

Proof. We proceed in three steps.

Step 1. Let us prove (3.1) and (3.2). Thanks to Lemma 5, we have $\sup _{\varepsilon \in E}\left(\left\|p_{\varepsilon}\right\|_{H^{1}\left(0, T ; L^{2}(\Omega)\right)}\right)<\infty$, and $\sup _{\varepsilon \in E}\left(\left\|u_{\varepsilon}^{i}\right\|_{H^{2}\left(0, T ; L^{2}(\Omega)\right)}\right)<$ $\infty(i=1,2,3)$. Hence, by Lemmas 1,3 and 4 , there exist a subsequence $E^{\prime} \subset E$ and functions $p_{0} \in H^{1}\left(0, T ; L^{2}\left(\Omega ; L_{p e r}^{2}(Y)\right)\right)$, $p_{1} \in L^{2}\left(Q ; L_{p e r}^{2}\left(Y ; H_{\#}^{1}(\Theta)\right)\right), u_{0}^{i} \in H^{2}\left(0, T ; L^{2}\left(\Omega ; L_{p e r}^{2}(Y)\right)\right)$ and $u_{1}^{i} \in$ $L^{2}\left(Q ; L_{\text {per }}^{2}\left(Y ; H_{\#}^{2}(\Theta)\right)\right)$ such that, as $E^{\prime} \ni \varepsilon \rightarrow 0$,

$$
\begin{aligned}
& \int_{Q} p_{\varepsilon} \Phi^{\varepsilon} d x d t \rightarrow \iint_{Q \times Z} p_{0} \Phi d x d t d y d \tau \\
& \int_{Q} \frac{\partial p_{\varepsilon}}{\partial t} \Phi^{\varepsilon} d x d t \rightarrow \iint_{Q \times Z}\left(\frac{\partial p_{0}}{\partial t}+\frac{\partial p_{1}}{\partial \tau}\right) \Phi d x d t d y d \tau, \\
& \int_{Q} u_{\varepsilon}^{i} \Phi^{\varepsilon} d x d t \rightarrow \iint_{Q \times Z} u_{0}^{i} \Phi d x d t d y d \tau, \\
& \int_{Q} \frac{\partial u_{\varepsilon}^{i}}{\partial t} \Phi^{\varepsilon} d x d t \rightarrow \iint_{Q \times Z} \frac{\partial u_{0}^{i}}{\partial t} \Phi d x d t d y d \tau, \\
& \int_{Q} \frac{\partial^{2} u_{\varepsilon}^{i}}{\partial t^{2}} \Phi^{\varepsilon} d x d t \rightarrow \iint_{Q \times Z}\left(\frac{\partial^{2} u_{0}^{i}}{\partial t^{2}}+\frac{\partial^{2} u_{1}^{i}}{\partial \tau^{2}}\right) \Phi d x d t d y d \tau
\end{aligned}
$$

( $i=1,2,3)$ for all $\Phi \in L^{2}\left(Q ; \mathcal{C}_{\text {per }}(Z)\right)$. Hence, (3.1) and (3.2) are the third line and the first line of (3.6) (respectively).

Step 2. Let us prove (3.3). By Lemma 5 we have

$$
\sup _{\varepsilon \in E}\left(\left\|\varepsilon \frac{\partial u_{\varepsilon}^{i}}{\partial x_{j}}\right\|_{L^{2}(Q)}\right)<\infty, \text { and } \sup _{\varepsilon \in E}\left(\left\|\varepsilon \frac{\partial^{2} u_{\varepsilon}^{i}}{\partial x_{j} \partial t}\right\|_{L^{2}(Q)}\right)<\infty(i, j=1,2,3) .
$$

Then according to (3.6), we conclude by Lemma 2 that

$$
\begin{aligned}
& \int_{Q} \varepsilon \frac{\partial u_{\varepsilon}^{i}}{\partial x_{j}} \varphi \psi^{\varepsilon} \theta^{\varepsilon} d x d t \rightarrow \iiint_{Q \times Y \times \Theta} \frac{\partial u_{0}^{i}}{\partial y_{j}} \varphi \otimes \psi \otimes \theta d x d t d y d \tau \\
& \int_{Q} \varepsilon \frac{\partial^{2} u_{\varepsilon}^{i}}{\partial x_{j} \partial t} \varphi \psi^{\varepsilon} \theta^{\varepsilon} d x d t \rightarrow \iiint_{Q \times Y \times \Theta} \frac{\partial^{2} u_{0}^{i}}{\partial y_{j} \partial t} \varphi \otimes \psi \otimes \theta d x d t d y d \tau
\end{aligned}
$$

$(i, j=1,2,3)$ for all $\varphi \in \mathcal{D}(Q), \psi \in \mathcal{C}_{\text {per }}^{\infty}(Y)$ and $\theta \in \mathcal{C}_{\text {per }}(\Theta)$; hence $u_{0}$, $\frac{\partial u_{0}}{\partial t} \in L^{2}\left(Q ; \mathbb{H}_{p e r}^{1}(Y)\right)$, where $u_{0}=\left(u_{0}^{i}\right) \equiv\left(u_{0}^{i}\right)_{i=1,2,3}$. Let us bear in mind 
that the preceding two limits give

$$
\begin{aligned}
& \int_{Q} \varepsilon E_{i j}\left(u_{\varepsilon}\right) \varphi \psi^{\varepsilon} \theta^{\varepsilon} d x d t \rightarrow \iint_{Q \times Y} e_{i j}\left(u_{0}\right) \varphi \otimes \psi d x d t d y \\
& \int_{Q} \varepsilon E_{i j}\left(\frac{\partial u_{\varepsilon}}{\partial t}\right) \varphi \psi^{\varepsilon} \theta^{\varepsilon} d x d t \rightarrow \iint_{Q \times Y} e_{i j}\left(\frac{\partial u_{0}}{\partial t}\right) \varphi \otimes \psi d x d t d y
\end{aligned}
$$

$(i, j=1,2,3)$ for all $\varphi \in \mathcal{D}(Q), \psi \in \mathcal{C}_{\text {per }}^{\infty}(Y)$ and $\theta \in \mathcal{C}_{\text {per }}(\Theta)$ with $\int_{\Theta} \theta d \tau=1$.

Since $E_{i j}\left(u_{\varepsilon}\right)=E_{i j}\left(\frac{\partial u_{\varepsilon}}{\partial t}\right)=0(i, j=1,2,3)$ and $p_{\varepsilon}=\frac{\partial p_{\varepsilon}}{\partial t}=0$ in $\Omega_{s}^{\varepsilon} \times(0, T)$ (see the definition of $V_{\varepsilon}$ ), we take in the first line in (3.6) and in (3.7) $\psi=\chi_{s} w$ where $w \in \mathcal{C}_{\text {per }}(Y)$ and $\chi_{s}$ is the characteristic function of $\widetilde{Y}_{s}$ defined on $\mathbb{R}_{y}^{3}$ [so that, as in $\left.(2.1), \varphi \otimes \psi \otimes \theta \in \mathcal{C}\left(\bar{Q} ; L_{p e r}^{\infty}(Z)\right)\right]$. It follows that

$$
\begin{aligned}
& \iint_{Q \times Y} \chi_{s} e_{i j}\left(u_{0}\right) \varphi \otimes w d x d t d y=0, \\
& \iint_{Q \times Y} \chi_{s} e_{i j}\left(\frac{\partial u_{0}}{\partial t}\right) \varphi \otimes w d x d t d y=0, \\
& \iint_{Q \times Y} \chi_{s} p_{0} \varphi \otimes w d x d t d y=0
\end{aligned}
$$

for all $\varphi \in \mathcal{D}(Q)$ and all $w \in \mathcal{C}_{\text {per }}(Y)$; and (3.3) is deduced.

Step 3. Let us prove (3.4) and (3.5). The first and second lines in (3.6) combining with (3.7) yield

$$
\begin{aligned}
& \iint_{Q \times Y} e_{i i}\left(u_{0}\right) \varphi \otimes w d x d t d y=0, \\
& \iint_{Q \times Y} e_{i i}\left(\frac{\partial u_{0}}{\partial t}\right) \varphi \otimes w d x d t d y=0
\end{aligned}
$$

for all $\varphi \in \mathcal{D}(Q), w \in \mathcal{C}_{\text {per }}^{\infty}(Y)$; and (3.4) follows. Furthermore, it follows to the first and third lines in (3.6) combining with (3.3), that

$$
\int_{Q}\left(\int_{Y_{f}} p_{0} d y\right) \varphi d x d t=\vartheta \int_{Q}\left(\int_{Y} u_{0}^{i} d y\right) \frac{\partial \varphi}{\partial x_{i}} d x d t
$$

for all $\varphi \in \mathcal{D}(Q)$, and (3.5) is proved.

Let us verify that $u_{0}$ satisfies the homogeneous initial conditions. Precisely we have the following lemma.

Lemma 7. $u_{0}, \frac{\partial u_{0}}{\partial t} \in \mathcal{C}\left([0, T] ; L^{2}\left(\Omega ; \mathbb{L}_{p e r}^{2}(Y)\right)\right)$ with $u_{0}(0)=\frac{\partial u_{0}}{\partial t}(0)=\omega$.

Proof. By Lemma $6, u_{0}$ and $\frac{\partial u_{0}}{\partial t}$ belong to $\mathcal{C}\left([0, T] ; L^{2}\left(\Omega ; \mathbb{L}_{p e r}^{2}(Y)\right)\right)$, so that $u_{0}(0)$ and $\frac{\partial u_{0}}{\partial t}(0)$ are well defined. Now, denoting by $[\cdot, \cdot]$ the 
scalar product in $L^{2}\left(\Omega ; \mathbb{L}_{p e r}^{2}(Y)\right)$, if $v \in \mathcal{C}_{T}^{1}\left([0, T] ; L^{2}\left(\Omega ; \mathbb{L}_{\text {per }}^{2}(Y)\right)\right)=\{w \in$ $\left.\mathcal{C}^{1}\left([0, T] ; L^{2}\left(\Omega ; \mathbb{L}_{\text {per }}^{2}(Y)\right)\right): w(T)=\omega\right\}$ then we have

$$
\int_{0}^{T}\left[u_{0}(t), v^{\prime}(t)\right] d t=-\int_{0}^{T}\left[u_{0}^{\prime}(t), v(t)\right] d t-\left[u_{0}(0), v(0)\right] .
$$

On the other hand, recalling that $u_{\varepsilon}$ and $\frac{\partial u_{\varepsilon}}{\partial t}$ lie in $L^{2}\left(0, T ; \mathbb{H}_{0}^{1}(\Omega)\right)$ with $u_{\varepsilon}(0)=\omega$, if $\beta \in \mathcal{C}_{T}^{1}([0, T])=\left\{\beta \in \mathcal{C}^{1}([0, T]): \beta(T)=0\right\}$ and $\psi \in L^{2}\left(\Omega ; \mathcal{C}_{\text {per }}(Y)^{3}\right)$ it follows that

$$
\int_{Q} \frac{\partial u_{\varepsilon}}{\partial t} \psi^{\varepsilon} \beta d x d t=-\int_{Q} u_{\varepsilon} \psi^{\varepsilon} \beta^{\prime} d x d t .
$$

The passage to the limit, as $E^{\prime} \ni \varepsilon \rightarrow 0$, permits to deduce

$$
\int_{0}^{T}\left[u_{0}^{\prime}(t), \psi\right] \beta(t) d t=-\int_{0}^{T}\left[u_{0}(t), \psi\right] \beta^{\prime}(t) d t .
$$

Using the density of $L^{2}\left(\Omega ; \mathcal{C}_{p e r}(Y)^{3}\right)$ in $L^{2}\left(\Omega ; \mathbb{L}_{p e r}^{2}(Y)\right)$, together with (3.8) we conclude that $\left[u_{0}(0), \varphi\right] \beta(0)=0$ for all $\varphi \in L^{2}\left(\Omega ; \mathbb{L}_{p e r}^{2}(Y)\right)$ and all $\beta \in \mathcal{C}_{T}^{1}([0, T])$. Therefore, we have $u_{0}(0)=\omega$. The same arguments give $\frac{\partial u_{0}}{\partial t}(0)=\omega$.

The following lemma will permit to achieve one part of the proof of Lemma 9.

Lemma 8. Let $S=\left(S^{i}\right) \in \mathcal{D}^{\prime}(Q)^{3} \quad\left(\mathcal{D}^{\prime}(Q)\right.$ being the space of distributions on $Q)$. Assume that

$$
\left(\int_{Y_{f}} w^{i} d y\right) S^{i} \in L^{2}(Q) \text { for all } w=\left(w^{i}\right) \in \mathcal{C}_{p e r}^{\infty}\left(Y_{f}\right)^{3}, \operatorname{div}_{y} w=0 .
$$

Then

$$
S=\left(S^{i}\right) \in \mathbb{L}^{2}(Q),
$$

where $\mathcal{C}_{\text {per }}^{\infty}\left(Y_{f}\right)$ denotes the space of functions in $\mathcal{C}_{\text {per }}^{\infty}(Y)$ with support contained in $\widetilde{Y}_{f}$ (see [11]).

Proof. We proceed in two steps.

Step 1. Let us prove the following: if $\xi=\left(\xi_{1}, \xi_{2}, \xi_{3}\right) \in \mathbb{R}^{3}$ with $\xi \neq \omega$, then there is $w=\left(w^{i}\right) \in \mathcal{C}_{p e r}^{\infty}\left(Y_{f}\right)^{3}, \operatorname{div}_{y} w=0$ such that $\xi_{i} \int_{Y_{f}} w^{i} d y \neq 0$, 
or equivalently if $\xi=\left(\xi_{1}, \xi_{2}, \xi_{3}\right) \in \mathbb{R}^{3}$ such that

$$
\xi_{i} \int_{Y_{f}} w^{i} d y=0 \text { for all } w=\left(w^{i}\right) \in \mathcal{C}_{\text {per }}^{\infty}\left(Y_{f}\right)^{3}, \operatorname{div}_{y} w=0
$$

then $\xi=\omega$.

Let us consider a point $\xi=\left(\xi_{1}, \xi_{2}, \xi_{3}\right) \in \mathbb{R}^{3}$ having property (3.11). There is $\psi \in H_{p e r}^{1}\left(Y_{f}\right)$ such that $\frac{\partial \psi}{\partial y_{i}}=\xi_{i}(i=1,2,3)$. Let $\chi$ be a polynomial function on $\tilde{Y}_{f}$ defined by $\chi(y)=\xi_{1} y_{1}+\xi_{2} y_{2}+\xi_{3} y_{3}$ for all $y=\left(y_{1}, y_{2}, y_{3}\right) \in \widetilde{Y}_{f}$. There exists a constant $c \in \mathbb{R}$ such that $\psi=\chi+c$ in $\tilde{Y}_{f}$. By virtue of periodicity of $\psi$, such equality is possible only if $\xi_{1}=\xi_{2}=\xi_{3}=0$.

Step 2. Now, let us prove (3.10). In what follows we assume there is $S=\left(S^{i}\right) \in \mathcal{D}^{\prime}(Q)^{3}$ satisfying (3.9).

Let $\xi=(1,0,0)$. According to the above step, there is $w_{1}=\left(w_{1}^{i}\right) \in$ $\mathcal{C}_{\text {per }}^{\infty}\left(Y_{f}\right)^{3}, \operatorname{div}_{y} w_{1}=0$ such that

$$
\int_{Y_{f}} w_{1}^{1} d y \neq 0
$$

We put

$$
a_{i}=\int_{Y_{f}} w_{1}^{i} d y \quad(i=1,2,3)
$$

By virtue of (3.9), there is $q_{1} \in L^{2}(Q)$ such that

$$
a_{1} S^{1}+a_{2} S^{2}=q_{1}-a_{3} S^{3} .
$$

Next let $\xi=\left(-a_{2}, a_{1}, 0\right)$. We have $a_{1} \neq 0$, then $\xi \neq \omega$, so that there is $w_{2}=\left(w_{2}^{i}\right) \in \mathcal{C}_{\text {per }}^{\infty}\left(Y_{f}\right)^{3}, \operatorname{div}_{y} w_{2}=0$ such that

$$
-a_{2} \int_{Y_{f}} w_{2}^{1} d y+a_{1} \int_{Y_{f}} w_{2}^{2} d y \neq 0 .
$$

By letting

$$
b_{i}=\int_{Y_{f}} w_{2}^{i} d y \quad(i=1,2,3)
$$

we have $a_{1} b_{2}-a_{2} b_{1} \neq 0$ and, according to (3.9), there is $q_{2} \in L^{2}(Q)$ such that

$$
b_{1} S^{1}+b_{2} S^{2}=q_{2}-b_{3} S^{3} .
$$


Hence, we are led to consider the system of linear equations (3.12)-(3.13). Since $a_{1} b_{2}-a_{2} b_{1} \neq 0$, then $S^{1}$ and $S^{2}$ are uniquely determined by

$$
S^{1}=\lambda_{1} q_{1}+\lambda_{2} q_{2}+\lambda_{3} S^{3} \text { and } S^{2}=\mu_{1} q_{1}+\mu_{2} q_{2}+\mu_{3} S^{3}
$$

$\lambda_{i}, \mu_{i}(i=1,2,3)$ being well defined.

Finally let $\xi=\left(\lambda_{3}, \mu_{3}, 1\right)$. There is $w_{3}=\left(w_{3}^{i}\right) \in \mathcal{C}_{p e r}^{\infty}\left(Y_{f}\right)^{3}, \operatorname{div}_{y} w_{3}=0$, such that

$$
\lambda_{3} \int_{Y_{f}} w_{3}^{1} d y+\mu_{3} \int_{Y_{f}} w_{3}^{2} d y+\int_{Y_{f}} w_{3}^{3} d y \neq 0 .
$$

We put

$$
c_{i}=\int_{Y_{f}} w_{3}^{i} d y \quad(i=1,2,3) .
$$

Then,

$$
\lambda_{3} c_{1}+\mu_{3} c_{2}+c_{3} \neq 0 .
$$

According to (3.9), there is $q_{3} \in L^{2}(Q)$ such that

$$
c_{1} S^{1}+c_{2} S^{2}+c_{3} S^{3}=q_{3} .
$$

By substituting (3.14), we have

$$
\left(\lambda_{3} c_{1}+\mu_{3} c_{2}+c_{3}\right) S^{3}=q_{3}-\left(\lambda_{2} c_{1}+\mu_{2} c_{2}\right) q_{2}-\left(\lambda_{1} c_{1}+\mu_{1} c_{2}\right) q_{1} .
$$

Thanks to (3.15), $S^{3}$ is determined as a linear combination of $q_{1}, q_{2}$, $q_{3} \in L^{2}(Q)$, that is $S^{3} \in L^{2}(Q)$. Therefore $S^{1}, S^{2} \in L^{2}(Q)$ by virtue of (3.13).

This being so, we will need the following lemma to derive the homogenized problem.

Lemma 9. By letting

$$
p(x, t)=-\frac{\vartheta}{\left|Y_{f}\right|} \operatorname{div}\left(\int_{Y} u_{0}(x, t, y) d y\right) \quad((x, t) \in Q),
$$

where $\left|Y_{f}\right|$ is the Lebesgue measure of $Y_{f}$, for almost all $(x, t) \in Q$ we have

$$
p_{0}(x, t, \cdot)=0 \text { in } Y_{s} \text { and } p(x, t) \text { in } Y_{f} .
$$

Furthermore

$$
\frac{\partial p}{\partial x_{i}} \in L^{2}\left(0, T ; L^{2}(\Omega)\right), i=1,2,3
$$


and the function $u_{1}=\left(u_{1}^{i}\right)$ in (3.6) verifies

$$
\int_{Y_{f}} u_{1}^{i}(x, t, y, \cdot) d y=\int_{Y_{s}} u_{1}^{i}(x, t, y, \cdot) d y=0, i=1,2,3 .
$$

Proof. Let us prove (3.17). We take in (1.2) test functions of the form $v=\varepsilon \varphi w^{\varepsilon} \phi(t) \theta^{\varepsilon}(t)$ for $0<t<T, \varepsilon \in E^{\prime}$, with $\varphi \in \mathcal{D}(\Omega), \phi \in \mathcal{D}((0, T))$, $\theta \in \mathcal{C}_{\text {per }}(\Theta), w=\left(w_{1}, w_{2}, w_{3}\right)$ each $w^{i}$ belonging to $\mathcal{C}_{\text {per }}^{\infty}\left(Y_{f}\right)$ [11]; next we integrate from 0 to $T$ and it follows that

$$
\begin{aligned}
& \varepsilon \int_{Q} \rho^{\varepsilon} \frac{\partial^{2} u_{\varepsilon}}{\partial t^{2}} \varphi w^{\varepsilon} \phi \theta^{\varepsilon} d x d t+\varepsilon \int_{Q} b_{i j k h} E_{k h}\left(\varepsilon \frac{\partial u_{\varepsilon}}{\partial t}\right)\left(e_{i j}(w)\right)^{\varepsilon} \varphi \phi \theta^{\varepsilon} d x d t \\
& -\varepsilon \int_{Q} p_{\varepsilon}(\operatorname{div} \varphi w)^{\varepsilon} \phi \theta^{\varepsilon} d x d t+\varepsilon^{2} \int_{Q} b_{i j k h} E_{k h}\left(\varepsilon \frac{\partial u_{\varepsilon}}{\partial t}\right)\left(E_{i j}(\varphi w)\right)^{\varepsilon} \phi \theta^{\varepsilon} d x d t \\
& -\varepsilon \int_{Q} f \varphi \phi w^{\varepsilon} \theta^{\varepsilon} d x d t=\int_{Q} p_{\varepsilon} \varphi\left(\operatorname{div}_{y} w\right)^{\varepsilon} \phi \theta^{\varepsilon} d x d t
\end{aligned}
$$

Passing to the limit, as $E^{\prime} \ni \varepsilon \rightarrow 0$, it follows by (3.6)-(3.7) that

$$
\iint_{Q \times Y_{f}} p_{0} \operatorname{div}_{y} w \varphi \phi d x d t d y=0 .
$$

This yields

$$
\int_{Y_{f}} p_{0}(x, t, \cdot) \frac{\partial \psi}{\partial y_{i}} d y=0 \text { a.e. in } \mathrm{Q} \text { for all } \psi \in \mathcal{C}_{p e r}^{\infty}\left(Y_{f}\right)(1 \leq i \leq 3) .
$$

Thus $p_{0}(x, t, \cdot)$ is constant in $Y_{f}$. Taking into account (3.3) and (3.5), we have drived (3.17).

Now, let us prove (3.18) and (3.19). We select in (1.2) test functions of the form $v=\varphi w^{\varepsilon} \phi(t) \theta^{\varepsilon}(t), t \in(0, T)$, with $\varphi \in \mathcal{D}(\Omega), \phi \in \mathcal{D}((0, T))$, $w \in \mathcal{C}_{\text {per }}^{\infty}\left(Y_{f}\right)^{3}, \operatorname{div}_{y} w=0$ and $\theta \in \mathcal{C}_{\text {per }}(\Theta)$; next we integrate from 0 to $T$ and obtain

$$
\begin{aligned}
& \int_{Q} \rho^{\varepsilon} \frac{\partial^{2} u_{\varepsilon}}{\partial t^{2}} \varphi w^{\varepsilon} \phi \theta^{\varepsilon} d x d t+\int_{Q} b_{i j k h} E_{k h}\left(\varepsilon \frac{\partial u_{\varepsilon}}{\partial t}\right)\left(e_{i j}(w)\right)^{\varepsilon} \varphi \phi \theta^{\varepsilon} d x d t \\
& -\int_{Q} p_{\varepsilon}(\operatorname{div}(\varphi w))^{\varepsilon} \phi \theta^{\varepsilon} d x d t+\varepsilon \int_{Q} b_{i j k h} E_{k h}\left(\varepsilon \frac{\partial u_{\varepsilon}}{\partial t}\right)\left(E_{i j}(\varphi w)\right)^{\varepsilon} \phi \theta^{\varepsilon} d x d t \\
& =\int_{Q} f \varphi w^{\varepsilon} \phi \theta^{\varepsilon} d x d t .
\end{aligned}
$$


Further we pass to the limit, as $E^{\prime} \ni \varepsilon \rightarrow 0$. Using Lemma 6 and property (3.17), we are led to

$$
\begin{aligned}
& \left(\int_{\Theta} \theta d \tau\right) \int_{Q}\left[\int_{Y_{f}}\left(\rho_{f} \frac{\partial^{2} u_{0}}{\partial t^{2}} \cdot w+\mu_{i j k h} e_{k h}\left(\frac{\partial u_{0}}{\partial t}\right) e_{i j}(w)-f \cdot w\right) d y\right] \varphi \phi d x d t \\
& +\iiint_{Q \times Y_{f} \times \Theta} \rho_{f} \frac{\partial^{2} u_{1}}{\partial \tau^{2}} \cdot w \varphi \phi \theta d x d t d y d \tau \\
& =\left(\int_{\Theta} \theta d \tau\right)\left(\int_{Y_{f}} w^{i} d y\right) \int_{Q} p(x, t) \frac{\partial \varphi}{\partial x_{i}}(x) \phi(t) d x d t
\end{aligned}
$$

for all $\varphi \in \mathcal{D}(\Omega), \phi \in \mathcal{D}((0, T)), w \in \mathcal{C}_{\text {per }}^{\infty}\left(Y_{f}\right)^{3}, \operatorname{div}_{y} w=0$ and $\theta \in \mathcal{C}_{p e r}(\Theta)$, where $\mu_{i j k h}=\mu\left(\delta_{i k} \delta_{j h}+\delta_{i h} \delta_{j k}\right)$. Taking $\theta=1$, we are drived to

$$
\left(\int_{Y_{f}} w^{i} d y\right) \frac{\partial p}{\partial x_{i}} \in L^{2}(Q) \quad \text { for all } w \in \mathcal{C}_{p e r}^{\infty}\left(Y_{f}\right)^{3}, \operatorname{div}_{y} w=0
$$

with

$$
\begin{aligned}
& \left(\int_{Y_{f}} w^{i} d y\right) \frac{\partial p}{\partial x_{i}}=\int_{Y_{f}}\left(\rho_{f} \frac{\partial^{2} u_{0}}{\partial t^{2}} \cdot w+\mu_{i j k h} e_{k h}\left(\frac{\partial u_{0}}{\partial t}\right) e_{i j}(w)-f \cdot w\right) d y \\
& (i=1,2,3) .
\end{aligned}
$$

Hence, taking into account Lemma 8 , we have $\frac{\partial p}{\partial x_{i}} \in L^{2}(Q), i=1,2,3$; and (3.18) follows.

Consequently $\iiint_{Q \times Y_{f} \times \Theta} \rho_{f} \frac{\partial^{2} u_{1}}{\partial \tau^{2}} \cdot w \varphi \phi \theta d x d t d y d \tau=0$ for all $\varphi \in \mathcal{D}(\Omega)$, $\phi \in \mathcal{D}((0, T)), w \in \mathcal{C}_{\text {per }}^{\infty}\left(Y_{f}\right)^{3}, \operatorname{div}_{y} w=0$ and $\theta \in \mathcal{C}_{\text {per }}(\Theta)$. Therefore $\frac{\partial^{2}}{\partial \tau^{2}} \int_{Y_{f}} u_{1}^{i} d y=0$ in $Q \times \Theta(i=1,2,3)$; that is for almost all $(x, t) \in Q$ the function $\frac{\partial}{\partial \tau} \int_{Y_{f}} u_{1}^{i}(x, t, y, \cdot) d y$ is constant with respect to $\tau$. By periodicity with respect to $\tau, \int_{Y_{f}} u_{1}^{i}(x, t, y, \cdot) d y=\lambda^{i}\left(\lambda^{i} \in \mathbb{R}\right)$. But $\int_{\Theta} u_{1}^{i} d \tau=0$, then we have $\int_{Y_{f}} u_{1}^{i}(x, t, y, \cdot) d y=0, i=1,2,3$.

Finally, we select in (1.2) test functions of the form $v=\varphi w^{\varepsilon} \phi(t) \theta^{\varepsilon}(t)$, $t \in(0, T)$, with $\varphi \in \mathcal{D}(\Omega), \phi \in \mathcal{D}((0, T))$, w constant in $Y$ and $\theta \in \mathcal{C}_{\text {per }}(\Theta)$; and we obtain, at the limit when $E^{\prime} \ni \varepsilon \rightarrow 0$

$$
\iint_{Q \times Y \times \Theta} \rho\left(\frac{\partial^{2} u_{0}^{i}}{\partial t^{2}}+\frac{\partial^{2} u_{1}^{i}}{\partial \tau^{2}}\right) \varphi \phi \theta d x d t d y d \tau=\iint_{Q \times \Theta}\left(f \varphi+p \frac{\partial \varphi}{\partial x_{i}}\right) \phi \theta d x d t d \tau
$$


$i=1,2,3$. Since

$$
\iint_{Q \times Y} \rho \frac{\partial^{2} u_{0}^{i}}{\partial t^{2}} \varphi \phi d x d t d y=\int_{Q}\left(f \varphi+p \frac{\partial \varphi}{\partial x_{i}}\right) \phi d x d t, i=1,2,3
$$

then $\iiint_{Q \times Y \times \Theta} \rho \frac{\partial^{2} u_{1}^{i}}{\partial \tau^{2}} \varphi \phi \theta d x d t d y d \tau=0$. Therefore $\int_{Y_{s}} u_{1}^{i}(x, t, y, \cdot) d y=$ $0, i=1,2,3$.

3.2 A suitable variational equation. Let us first prove a general result.

Lemma 10. Let $\Omega_{0}$ and $\Omega_{1}$ be two smooth open bounded sets in $\mathbb{R}^{N}$ $(N \geq 1)$ with the property

$$
\left.\bar{\Omega}_{0} \subset \Omega_{1} \quad \bar{\Omega}_{0} \text { being the closure of } \Omega_{0} \text { in } \mathbb{R}^{N}\right) \text {, }
$$

and let $\gamma_{\circ} \in \mathcal{L}\left(H^{1}\left(\Omega_{0}\right) ; H^{\frac{1}{2}}\left(\partial \Omega_{0}\right)\right)$, the usual trace operator on $\Omega_{0}$. Then there exist two continuous linear mappings $\Gamma_{\circ} \in \mathcal{L}\left(H_{0}^{1}\left(\Omega_{1}\right) ; H^{\frac{1}{2}}\left(\partial \Omega_{0}\right)\right)$ and $R_{\circ} \in \mathcal{L}\left(H^{\frac{1}{2}}\left(\partial \Omega_{0}\right) ; H_{0}^{1}\left(\Omega_{1}\right)\right)\left(H_{0}^{1}\left(\Omega_{1}\right)\right.$ endowed with the $H^{1}\left(\Omega_{1}\right)$-norm) such that

$$
\begin{array}{ll}
\left(\Gamma_{\circ} \circ R_{\circ}\right) g=g & \text { for all } g \in H^{\frac{1}{2}}\left(\partial \Omega_{0}\right), \text { and } \\
\left(R_{\circ} \circ \Gamma_{\circ}\right) v=v & \text { for all } v \in\left(\operatorname{ker} \Gamma_{\circ}\right)^{\perp}
\end{array}
$$

where $\left(\operatorname{ker} \Gamma_{\circ}\right)^{\perp}$ is the orthogonal in $H_{0}^{1}\left(\Omega_{1}\right)$ of the kernel of $\Gamma_{\circ}$, ० denotes usual composition, $\mathcal{L}\left(H_{0}^{1}\left(\Omega_{1}\right) ; H^{\frac{1}{2}}\left(\partial \Omega_{0}\right)\right.$ being the vector space of continuous linear mappings from $H_{0}^{1}\left(\Omega_{1}\right)$ into $H^{\frac{1}{2}}\left(\partial \Omega_{0}\right)$, and $\mathcal{L}\left(H^{\frac{1}{2}}\left(\partial \Omega_{0}\right) ; H_{0}^{1}\left(\Omega_{1}\right)\right)$ being that of continuous linear mappings from $H^{\frac{1}{2}}\left(\partial \Omega_{0}\right)$ into $H_{0}^{1}\left(\Omega_{1}\right)$.

Proof. By noting $\left.v\right|_{\Omega_{0}}$ the restriction of $v$ on $\Omega_{0}$, we construct a surjective mapping $\Gamma_{\circ}$ by $\Gamma_{\circ} v=\gamma_{\circ}\left(\left.v\right|_{\Omega_{0}}\right) \quad\left(v \in H_{0}^{1}\left(\Omega_{1}\right)\right)$. It is an obvious matter to verify that $\Gamma_{\circ}$ belongs to $\mathcal{L}\left(H_{0}^{1}\left(\Omega_{1}\right) ; H^{\frac{1}{2}}\left(\partial \Omega_{0}\right)\right)$, where $H_{0}^{1}\left(\Omega_{1}\right)$ and $H^{\frac{1}{2}}\left(\partial \Omega_{0}\right)$ are endowed with the usual $H^{1}\left(\Omega_{1}\right)$-norm and $H^{\frac{1}{2}}\left(\partial \Omega_{0}\right)$ norm respectively. Using (3.20) and the construction of $\Gamma_{\circ}$, we prove easily the equality $H^{\frac{1}{2}}\left(\partial \Omega_{0}\right)=\left\{g \in L^{2}\left(\partial \Omega_{0}\right): \exists v \in H_{0}^{1}\left(\Omega_{1}\right)\right.$ with $\left.\Gamma_{\circ} v=g\right\}$. This being so, we endow $H^{\frac{1}{2}}\left(\partial \Omega_{0}\right)$ with the norm $\|g\|=\inf \left\{\|v\|_{H^{1}\left(\Omega_{1}\right)}: v \in\right.$ $H_{0}^{1}\left(\Omega_{1}\right)$ with $\left.\Gamma_{\circ} v=g\right\}\left(g \in H^{\frac{1}{2}}\left(\partial \Omega_{0}\right)\right)$ which makes it a Banach space. By a classical result of minimization of functionals, for fixed $g \in H^{\frac{1}{2}}\left(\partial \Omega_{0}\right)$ there exists a unique couple $\left(v_{\circ}, w_{\circ}\right) \in H_{0}^{1}\left(\Omega_{1}\right) \times H^{1}\left(\Omega_{0}\right)$ such that $g=\Gamma_{\circ} v_{\circ}=\gamma_{\circ} w_{\circ},\|g\|=\left\|v_{\circ}\right\|_{H^{1}\left(\Omega_{1}\right)}$ and $\|g\|_{H^{\frac{1}{2}}\left(\partial \Omega_{0}\right)}=\left\|w_{\circ}\right\|_{H^{1}\left(\Omega_{0}\right)}$. Since $\|g\|_{H^{\frac{1}{2}\left(\partial \Omega_{0}\right)}} \leq\left\|\left(\left.v_{\circ}\right|_{\Omega_{0}}\right)\right\|_{H^{1}\left(\Omega_{0}\right)}$, then we have $\|g\|_{H^{\frac{1}{2}\left(\partial \Omega_{0}\right)}} \leq\|g\|$; and by virtue of the open mapping theorem (see, e.g., [5]), it follows that both the 
norms $\|\cdot\|_{H^{\frac{1}{2}}\left(\partial \Omega_{0}\right)}$ and $\|\cdot\|$ are equivalent on $H^{\frac{1}{2}}\left(\partial \Omega_{0}\right)$. Finally, we apply the usual canonical decomposition of $\Gamma_{\circ}$ to define $R_{\circ}$ as a linear mapping from $H^{\frac{1}{2}}\left(\partial \Omega_{0}\right)$ into $H_{0}^{1}\left(\Omega_{1}\right)$ with $(3.21)$ and there are two constants $c_{\circ}, c_{1}$ such that $\left\|R_{\circ} g\right\|_{H^{1}\left(\Omega_{1}\right)} \leq c_{\circ}\|g\| \leq c_{\circ} c_{1}\|g\|_{H^{\frac{1}{2}}\left(\partial \Omega_{0}\right)}$ for all $g \in H^{\frac{1}{2}}\left(\partial \Omega_{0}\right)$.

Lemma 10 leads us to the following

Lemma 11. Let $\Omega_{0}$ and $\Omega_{1}$ be two smooth open bounded sets in $\mathbb{R}^{N}$ $(N \geq 1)$ with the property (3.20), let $\Phi_{\circ}$ be a linear form on $H_{0}^{1}\left(\Omega_{1}\right)$, and let $V$ be a fixed vector subspace of $H_{0}^{1}\left(\Omega_{1}\right)$. If

$$
\Phi_{\circ}=0 \text { in } V \cap \operatorname{ker} \Gamma_{\circ} \text {, }
$$

then

$$
\Phi_{\circ}=\Phi_{\circ} \circ R_{\circ} \circ \Gamma_{\circ} \text { in } V .
$$

Proof. Under the above assumptions, for any $v \in V, v=v_{\circ}+v_{1}$, $v_{\circ} \in\left(\operatorname{ker} \Gamma_{\circ}\right)^{\perp}$ and $v_{1} \in \operatorname{ker} \Gamma_{\circ}$, we have $\left(\Phi_{\circ} \circ R_{\circ} \circ \Gamma_{\circ}\right) v=\left(\Phi_{\circ} \circ R_{\circ} \circ \Gamma_{\circ}\right) v_{\circ}=$ $\Phi_{\circ} v_{\circ}=\Phi_{\circ} v$ (see (3.21) for the second equality).

We are now in position to prove that the solution $u_{\varepsilon}$ of (1.1)-(1.2) can be inserted in an appropriate variational furmulation. Indeed, it is not easy to take test functions in (1.1) of the form $v(x, t)=\varphi(x, t) w\left(\frac{x}{\varepsilon}\right) \theta\left(\frac{t}{\varepsilon}\right)$, $\varphi \in \mathcal{D}(Q), w \in \mathbb{H}_{\text {per }}^{1}(Y), \theta \in H_{\text {per }}^{1}(\Theta)$, which belong to $V_{\varepsilon}$. The following proposition is then fundamental in the rest of this section.

Proposition 1. There is $0<\varepsilon_{0} \leq 1$ such that for $0<\varepsilon \leq \varepsilon_{0}$, there exists $z_{\varepsilon} \in L^{\infty}\left(0, T ; \mathbb{H}^{1}\left(\Omega_{s}^{\varepsilon} ; \mathbb{R}\right)\right)$ verifying

$$
\begin{aligned}
& \int_{Q} \rho^{\varepsilon} \frac{\partial^{2} u_{\varepsilon}}{\partial t^{2}} \cdot v d x d t+\varepsilon^{2} \int_{Q} b_{i j k h} E_{k h}\left(\frac{\partial u_{\varepsilon}}{\partial t}\right) E_{i j}(v) d x d t \\
& (3.22)+\vartheta \int_{Q} \operatorname{div} u_{\varepsilon} \operatorname{div} v d x d t=\int_{Q_{s}^{\varepsilon}} E_{i j}\left(z_{\varepsilon}\right) E_{i j}(v) d x d t+\int_{Q} f \cdot v d x d t
\end{aligned}
$$

for all $v \in L^{2}\left(0, T ; \mathbb{H}_{0}^{1}(\Omega ; \mathbb{R})\right)$, where $u_{\varepsilon}$ is the solution of (1.1)-(1.2). Furthermore,

$$
\sup _{0<\varepsilon \leq \varepsilon_{0}}\left(\left\|E_{i j}\left(z_{\varepsilon}\right)\right\|_{L^{2}\left(0, T: L^{2}\left(\Omega_{s}^{\varepsilon}\right)\right)}\right)<\infty \quad(1 \leq i, j \leq 3) .
$$

Proof. We will proceed in five steps.

Step 1. Let us introduce $\Omega_{1}=\left\{x \in \mathbb{R}^{3}: d(x, \bar{\Omega})<1\right\}$, where $d$ designates the Euclidean metric and $\bar{\Omega}$ the closure of $\Omega$ in $\mathbb{R}_{x}^{3}$. For 
$0<\varepsilon \leq 1$, let $t_{\varepsilon}=\left\{k \in \mathbb{Z}^{3}: \varepsilon\left(k+Y_{s}\right) \subset \Omega_{1}\right\}$. Clearly there exists $0<\varepsilon_{0} \leq 1$ such that $t_{\varepsilon}$ is nonempty for $0<\varepsilon \leq \varepsilon_{0}$. By letting

$$
\Omega_{0}^{\varepsilon}=\bigcup_{k \in t_{\varepsilon}} \varepsilon\left(k+Y_{s}\right) \text { and } \partial \Omega_{0}^{\varepsilon}=\bigcup_{k \in t_{\varepsilon}} \varepsilon\left(k+\partial Y_{s}\right), 0<\varepsilon \leq \varepsilon_{0},
$$

the bounded sets $\Omega_{1}$ and $\Omega_{0}^{\varepsilon}$ are opened and at least of class $\mathcal{C}^{1}$ in $\mathbb{R}_{x}^{3}$ with $\bar{\Omega}_{0}^{\varepsilon} \subset \Omega_{1}$. Let $0<\varepsilon \leq \varepsilon_{0}$ be fixed, and let $u_{\varepsilon}$ be the function defined in Theorem 1. For each $0 \leq t \leq T$, the linear form $\Phi(\varepsilon, t)$ on $\mathbb{H}_{0}^{1}\left(\Omega_{1} ; \mathbb{R}\right)$ defined by

$$
\begin{aligned}
\Phi(\varepsilon, t)(v)= & \int_{\Omega} \rho^{\varepsilon} u_{\varepsilon}^{\prime \prime}(t) \cdot v d x+\varepsilon^{2} \int_{\Omega} b_{i j k h} E_{k h}\left(u_{\varepsilon}^{\prime}(t)\right) E_{i j}(v) d x \\
& +\vartheta \int_{\Omega} \operatorname{div} u_{\varepsilon}(t) \operatorname{div} v d x-\int_{\Omega} f(t) \cdot v d x \quad\left(v \in \mathbb{H}_{0}^{1}\left(\Omega_{1} ; \mathbb{R}\right)\right)
\end{aligned}
$$

is continuous.

Step 2. We want to decompose $\Phi(\varepsilon, t)$, further we will apply Lemma 11. Let $\gamma_{\circ}^{\varepsilon}$ be the trace operator on $\partial \Omega_{0}^{\varepsilon}$. Let $\Gamma_{\circ}^{\varepsilon}$ be the operator on $\mathbb{H}_{0}^{1}\left(\Omega_{1} ; \mathbb{R}\right)$ defined by $\Gamma_{\circ}^{\varepsilon} v=\gamma_{\circ}^{\varepsilon}\left(\left.v\right|_{\Omega_{0}^{\varepsilon}}\right)=\left(\gamma_{\circ}^{\varepsilon}\left(v^{i} \mid \Omega_{0}^{\varepsilon}\right)\right)\left(v=\left(v^{i}\right) \in \mathbb{H}_{0}^{1}\left(\Omega_{1} ; \mathbb{R}\right)\right)$, and let $R_{\circ}^{\varepsilon}$ be defined in the sense of Lemma 10. We put $\Phi(\varepsilon, t)=\Phi_{\circ}(\varepsilon, t)+\Phi_{1}(\varepsilon, t)$ and $\Upsilon(\varepsilon, t)=\Phi_{\circ}(\varepsilon, t) \circ R_{\circ}^{\varepsilon}$ (usual composition), where $\Phi_{\circ}(\varepsilon, t)$ and $\Phi_{1}(\varepsilon, t)$ are the continuous linear forms on $\mathbb{H}_{0}^{1}\left(\Omega_{1} ; \mathbb{R}\right)$ defined by

$$
\begin{aligned}
\Phi_{\circ}(\varepsilon, t)(v)= & \int_{\Omega_{f}^{\varepsilon}} \rho_{f} u_{\varepsilon}^{\prime \prime}(t) \cdot v d x+\varepsilon^{2} \int_{\Omega} b_{i j k h} E_{k h}\left(u_{\varepsilon}^{\prime}(t)\right) E_{i j}(v) d x \\
& +\vartheta \int_{\Omega} \operatorname{div} u_{\varepsilon}(t) \operatorname{div} v d x-\int_{\Omega_{f}^{\varepsilon}} f(t) \cdot v d x
\end{aligned}
$$

and

$$
\Phi_{1}(\varepsilon, t)(v)=\int_{\Omega_{s}^{\varepsilon}} \rho_{f} u_{\varepsilon}^{\prime \prime}(t) \cdot v d x-\int_{\Omega_{s}^{\varepsilon}} f(t) \cdot v d x
$$

for all $v \in \mathbb{H}_{0}^{1}\left(\Omega_{1} ; \mathbb{R}\right)$. It follows immediately that $\Upsilon(\varepsilon, t)$ is a continuous linear form on $\mathbb{H}^{\frac{1}{2}}\left(\partial \Omega_{0}^{\varepsilon}\right)$. By letting

$$
\begin{aligned}
& \widetilde{\mathbb{H}_{0}^{1}(\Omega)}=\left\{v \in \mathbb{H}_{0}^{1}\left(\Omega_{1} ; \mathbb{R}\right): v=\omega \text { in } \Omega_{1} \backslash \Omega\right\} \text { and } \\
& \widetilde{V}_{\varepsilon}=\left\{v \in \widetilde{\mathbb{H}_{0}^{1}(\Omega)}: E_{i j}(v)=0 \text { in } \Omega_{0}^{\varepsilon}(i, j=1,2,3)\right\},
\end{aligned}
$$

$\widetilde{\mathbb{H}_{0}^{1}(\Omega)}$ is a closed vector subspace of $\mathbb{H}_{0}^{1}\left(\Omega_{1}\right)$ and $\widetilde{V}_{\varepsilon}$ is that of $\widetilde{\mathbb{H}_{0}^{1}(\Omega)}$. Since $\Phi_{0}(\varepsilon, t)=0$ in $\widetilde{\mathbb{H}_{0}^{1}(\Omega)} \cap \operatorname{ker} \Gamma_{\circ}^{\varepsilon}$, Lemma 11 leads us to

$$
\Phi(\varepsilon, t)=\Upsilon(\varepsilon, t) \circ \Gamma_{\circ}^{\varepsilon}+\Phi_{1}(\varepsilon, t) \text { in } \widetilde{\mathbb{H}_{0}^{1}(\Omega)}
$$


with

$$
\Upsilon(\varepsilon, t) \circ \Gamma_{\circ}^{\varepsilon}+\Phi_{1}(\varepsilon, t)=0 \quad \text { in } \widetilde{V}_{\varepsilon}
$$

Step 3. We want to solve the variational problem (for fixed $t \in[0, T]$ and $\left.0<\varepsilon \leq \varepsilon_{0}\right)$ :

$$
\begin{aligned}
& \text { Find } z_{\varepsilon}(t) \in \mathbb{H}^{1}\left(\Omega_{s}^{\varepsilon} ; \mathbb{R}\right) \text { such that } \\
& \int_{\Omega_{s}^{\varepsilon}} E_{i j}\left(z_{\varepsilon}(t)\right) E_{i j}(v) d x=\Upsilon(\varepsilon, t)\left(\Gamma_{\circ}^{\varepsilon} v\right)+\Phi_{1}(\varepsilon, t)(v) \text { for } v \in \widetilde{\mathbb{H}_{0}^{1}(\Omega)}
\end{aligned}
$$

We begin by noting that the mapping $v \rightarrow\left(\int_{\Omega_{s}^{\varepsilon}} E_{i j}(v) E_{i j}(v) d x\right)^{\frac{1}{2}}$ is a norm in the closed vector subspace $\widetilde{\mathbb{H}^{1}\left(\Omega_{s}^{\varepsilon}\right)}=\left\{v \in \mathbb{H}^{1}\left(\Omega_{0}^{\varepsilon} ; \mathbb{R}\right): v=\omega\right.$ in $\left.\Omega_{0}^{\varepsilon} \backslash \Omega_{s}^{\varepsilon}\right\}$ of $\mathbb{H}^{1}\left(\Omega_{0}^{\varepsilon} ; \mathbb{R}\right)$, equivalent to the usual $\mathbb{H}^{1}\left(\Omega_{0}^{\varepsilon}\right)$-norm . Further, we decompose the space $\widetilde{\mathbb{H}^{1}\left(\Omega_{s}^{\varepsilon}\right)}$ as $\widetilde{\mathbb{H}^{1}\left(\Omega_{s}^{\varepsilon}\right)}=W_{\varepsilon} \oplus\left(\widetilde{\mathbb{H}^{1}\left(\Omega_{s}^{\varepsilon}\right)} / W_{\varepsilon}\right)$, where $W_{\varepsilon}=\left\{v \in \widetilde{\mathbb{H}^{1}\left(\Omega_{s}^{\varepsilon}\right)}: E_{i j}(v)=0(i, j=1,2,3)\right\}$ and $\widetilde{\mathbb{H}^{1}\left(\Omega_{s}^{\varepsilon}\right) / W_{\varepsilon}}=\{v \in$ $\widetilde{\mathbb{H}^{1}\left(\Omega_{s}^{\varepsilon}\right)}: \int_{\Omega_{s}^{\varepsilon}} u v d x=0$ for all $\left.u \in W_{\varepsilon}\right\}$. Let $v \in W_{\varepsilon}$ and $\widetilde{v}$ its extension on $\Omega_{1}$ such that $\widetilde{v}=\omega$ on $\Omega_{1} \backslash \Omega$. Then $\widetilde{v} \in \widetilde{V}_{\varepsilon}$ and (3.26) implies

$$
\Upsilon(\varepsilon, t)\left(\gamma_{\circ}^{\varepsilon} v\right)+\int_{\Omega_{s}^{\varepsilon}} u_{\varepsilon}^{\prime \prime}(t) \cdot v d x-\int_{\Omega_{s}^{\varepsilon}} f(t) \cdot v d x=0 \quad\left(v \in W_{\varepsilon}\right)
$$

with $\gamma_{\circ}^{\varepsilon} v=\Gamma_{\circ}^{\varepsilon} \widetilde{v}$. Since $\left\|\gamma_{\circ}^{\varepsilon} v\right\|_{\mathbb{H}^{1}\left(\partial \Omega_{0}^{\varepsilon}\right)} \leq\|\widetilde{v}\|_{\mathbb{H}^{1}\left(\Omega_{0}^{\varepsilon}\right)}$, the linear form

$$
v \rightarrow \Upsilon(\varepsilon, t)\left(\gamma_{\circ}^{\varepsilon} v\right)+\int_{\Omega_{s}^{\varepsilon}} u_{\varepsilon}^{\prime \prime}(t) \cdot v d x-\int_{\Omega_{s}^{\varepsilon}} f(t) \cdot v d x
$$

is continuous on $\widetilde{\mathbb{H}^{1}\left(\Omega_{s}^{\varepsilon}\right)}$. By virtue of (3.28), there exists a unique function $z_{\varepsilon}(t) \in \widetilde{\mathbb{H}^{1}\left(\Omega_{s}^{\varepsilon}\right)} / W_{\varepsilon}$ such that

$$
\int_{\Omega_{s}^{\varepsilon}} E_{i j}\left(z_{\varepsilon}(t)\right) E_{i j}(v) d x=\Upsilon(\varepsilon, t)\left(\gamma_{\circ}^{\varepsilon} v\right)+\int_{\Omega_{s}^{\varepsilon}} u_{\varepsilon}^{\prime \prime}(t) \cdot v d x-\int_{\Omega_{s}^{\varepsilon}} f(t) \cdot v d x
$$

for all $v \in \widetilde{\mathbb{H}^{1}\left(\Omega_{s}^{\varepsilon}\right)} / W_{\varepsilon}$. But, according to the definition of $W_{\varepsilon}$, we may replace the space of test functions $\widetilde{\mathbb{H}^{1}\left(\Omega_{s}^{\varepsilon}\right)} / W_{\varepsilon}$ by $\widetilde{\mathbb{H}^{1}\left(\Omega_{s}^{\varepsilon}\right)}$, next $\widetilde{\mathbb{H}^{1}\left(\Omega_{s}^{\varepsilon}\right)}$ is replaced by $\widetilde{\mathbb{H}_{0}^{1}(\Omega)}$ to obtain $(3.27)$.

Step 4. Let us prove that the function $z_{\varepsilon}: t \rightarrow z_{\varepsilon}(t)$ from $[0, T]$ into $\mathbb{H}^{1}\left(\Omega_{s}^{\varepsilon}\right)$ belongs to $L^{\infty}\left(0, T ; \mathbb{H}^{1}\left(\Omega_{s}^{\varepsilon}\right)\right)$ with $(3.23)$ : Let $t \in(0, T)$ and $h \in \mathbb{R}$ 
such that $t+h \in(0, T)$. According to (3.29) we have

$$
\begin{aligned}
\int_{\Omega_{s}^{\varepsilon}} E_{i j}\left(z_{\varepsilon}(t+h)-z_{\varepsilon}(t)\right) E_{i j}(v) d x= & (\Upsilon(\varepsilon, t+h)-\Upsilon(\varepsilon, t))\left(\gamma_{o}^{\varepsilon} v\right) \\
& +\int_{\Omega_{s}^{\varepsilon}}\left(u_{\varepsilon}^{\prime \prime}(t+h)-u_{\varepsilon}^{\prime \prime}(t)\right) \cdot v d x \\
& -\int_{\Omega_{s}^{\varepsilon}}(f(t+h)-f(t)) \cdot v d x .
\end{aligned}
$$

We take $v=z_{\varepsilon}(t+h)-z_{\varepsilon}(t)$, and it follows

$$
\begin{aligned}
c\left\|z_{\varepsilon}(t+h)-z_{\varepsilon}(t)\right\|_{\mathbb{H}^{1}\left(\Omega_{s}^{\varepsilon}\right)} \leq & \|\Upsilon(\varepsilon, t+h)-\Upsilon(\varepsilon, t)\|_{\mathbb{H}^{-\frac{1}{2}}\left(\partial \Omega_{0}^{\varepsilon}\right)} \\
& +\left\|u_{\varepsilon}^{\prime \prime}(t+h)-u_{\varepsilon}^{\prime \prime}(t)\right\|_{\mathbb{L}^{2}\left(\Omega_{s}^{\varepsilon}\right)} \\
& +\|f(t+h)-f(t)\|_{\mathbb{L}^{2}\left(\Omega_{s}^{\varepsilon}\right)}
\end{aligned}
$$

where $c$ does not depend to $t, h$ and $0<\varepsilon \leq \varepsilon_{0}$. But $\Upsilon(\varepsilon, t)=$ $\Phi_{\circ}(\varepsilon, t) \circ R_{\circ}^{\varepsilon}$, then there exists a constant $c$ independent to $t, h$ and $0<\varepsilon \leq \varepsilon_{0}$ such that

$$
\begin{aligned}
c\|\Upsilon(\varepsilon, t+h)-\Upsilon(\varepsilon, t)\|_{\mathbb{H}^{-\frac{1}{2}}\left(\partial \Omega_{0}^{\varepsilon}\right)} & \\
\leq & \left\|u_{\varepsilon}^{\prime \prime}(t+h)-u_{\varepsilon}^{\prime \prime}(t)\right\|_{\mathbb{L}^{2}\left(\Omega_{f}^{\varepsilon}\right)}+\varepsilon\left\|u_{\varepsilon}^{\prime}(t+h)-u_{\varepsilon}^{\prime}(t)\right\|_{\mathbb{H}^{1}(\Omega)} \\
& +\left\|\operatorname{div} u_{\varepsilon}(t+h)-\operatorname{div} u_{\varepsilon}(t)\right\|_{\mathbb{L}^{2}(\Omega)}+\|f(t+h)-f(t)\|_{\mathbb{L}^{2}\left(\Omega_{f}^{\varepsilon}\right)} .
\end{aligned}
$$

Thus, there is a constant $c>0$ independent to $t, h$ and $0<\varepsilon \leq \varepsilon_{0}$ such that

$$
\begin{aligned}
c\left\|z_{\varepsilon}(t+h)-z_{\varepsilon}(t)\right\|_{\mathbb{H}^{1}\left(\Omega_{s}^{\varepsilon}\right)} & \\
\leq & \left\|u_{\varepsilon}^{\prime \prime}(t+h)-u_{\varepsilon}^{\prime \prime}(t)\right\|_{\mathbb{L}^{2}(\Omega)}+\varepsilon\left\|u_{\varepsilon}^{\prime}(t+h)-u_{\varepsilon}^{\prime}(t)\right\|_{\mathbb{H}^{1}(\Omega)} \\
& +\left\|\operatorname{div} u_{\varepsilon}(t+h)-\operatorname{div} u_{\varepsilon}(t)\right\|_{\mathbb{L}^{2}(\Omega)}+\|f(t+h)-f(t)\|_{\mathbb{L}^{2}(\Omega)} .
\end{aligned}
$$

Since $f, u_{\varepsilon}, \operatorname{div} u_{\varepsilon}, u_{\varepsilon}^{\prime}$ and $u_{\varepsilon}^{\prime \prime}$ are measurable, then the function $z_{\varepsilon}: t \rightarrow$ $z_{\varepsilon}(t)$ from $[0, T]$ into $\mathbb{H}^{1}\left(\Omega_{s}^{\varepsilon}\right)$ is measurable. Furthermore, we are quickly drived to $\left\|z_{\varepsilon}(t)\right\|_{\widetilde{H^{1}\left(\Omega_{s}^{\varepsilon}\right)}} \leq c$ for almost all $t \in(0, T)$ and all $0<\varepsilon \leq \varepsilon_{0}$, which proves that $z_{\varepsilon}$ belongs to $L^{\infty}\left(0, T ; \mathbb{H}^{1}\left(\Omega_{s}^{\varepsilon}\right)\right)$ with (3.23).

Step 5. Let us derive (3.22). By combining (3.24), (3.25) and (3.27), we deduce (3.22).

We end this subsection by proving a crucial lemma for the choice of test functions. We denote by $R_{\text {per }}^{s}$, the space of $w \in \mathbb{H}_{\text {per }}^{1}\left(Y_{s} ; \mathbb{R}\right), e_{i j}(w)=0$, 
$i, j=1,2,3$ and by $\mathbb{H}_{\text {per }}^{1}\left(Y_{s} ; \mathbb{R}\right) / R_{\text {per }}^{s}$, the space of $u \in \mathbb{H}_{\text {per }}^{1}\left(Y_{s} ; \mathbb{R}\right)$, $\int_{Y_{s}} u \cdot w d y=0$ for all $w \in R_{p e r}^{s}$.

We have $\mathbb{H}_{\text {per }}^{1}\left(Y_{s} ; \mathbb{R}\right)=R_{\text {per }}^{s} \oplus\left(\mathbb{H}_{\text {per }}^{1}\left(Y_{s} ; \mathbb{R}\right) / R_{\text {per }}^{s}\right)$, and the seminorm

$$
u \rightarrow e_{s}(u)=\left(\int_{Y_{s}} e_{i j}(u) e_{i j}(u) d y\right)^{\frac{1}{2}}
$$

is a norm on $\mathbb{H}_{\text {per }}^{1}\left(Y_{s} ; \mathbb{R}\right) / R_{\text {per }}^{s}$ equivalent to the usual $\mathbb{H}^{1}\left(Y_{s}\right)$-norm. We endow $L^{2}\left(Q ; \mathbb{H}_{\text {per }}^{1}\left(Y_{s} ; \mathbb{R}\right) / R_{\text {per }}^{s}\right)$ with the hilbertian norm

$$
\|u\|_{Q, Y_{s}}=\left(\int_{Q}\left[e_{s}(u(x))\right]^{2} d x\right)^{\frac{1}{2}} \quad\left(u \in L^{2}\left(Q ; \mathbb{H}_{\text {per }}^{1}\left(Y_{s} ; \mathbb{R}\right) / R_{p e r}^{s}\right)\right) .
$$

Lemma 12. Given $\Psi_{0} \in H^{1}\left(Q ; \mathbb{H}_{p e r}^{1}(Y ; \mathbb{R})\right), e_{i j}\left(\Psi_{0}\right)=0$ in $Q \times Y_{s}$ $(i, j=1,2,3)$, there exists $\Psi_{1} \in L^{2}\left(Q ; \mathbb{H}_{\text {per }}^{1}\left(Y_{s} ; \mathbb{R}\right)\right)$ such that

$$
\iint_{Q \times Y_{s}} e_{i j}\left(\Psi_{1}\right) e_{i j}(v) d x d t d y=-\iint_{Q \times Y_{s}} E_{i j}\left(\Psi_{0}\right) e_{i j}(v) d x d t d y
$$

for all $v \in L^{2}\left(Q ; \mathbb{H}_{\text {per }}^{1}(Y ; \mathbb{R})\right)$.

Proof. There exists exactly one function $u_{1} \in L^{2}\left(Q ; \mathbb{H}^{1}\left(Y_{s} ; \mathbb{R}\right) / R^{s}\right)$ such that

$$
\iint_{Q \times Y_{s}} e_{i j}\left(u_{1}\right) e_{i j}(v) d x d t d y=-\iint_{Q \times Y_{s}} E_{i j}\left(\Psi_{0}\right) e_{i j}(v) d x d t d y
$$

for all $v \in L^{2}\left(Q ; \mathbb{H}^{1}\left(Y_{s} ; \mathbb{R}\right) / R^{s}\right)$, where $R^{s}=\left\{w \in \mathbb{H}^{1}\left(Y_{s} ; \mathbb{R}\right): e_{i j}(w)=0\right.$, $i, j=1,2,3\}$. Extending by periodicity, we obtain a unique function (still denoted $\left.u_{1}\right) u_{1} \in L^{2}\left(Q ; \mathbb{H}_{\text {per }}^{1}\left(Y_{s} ; \mathbb{R}\right) / R_{\text {per }}^{s}\right)$ such that

$$
\iint_{Q \times Y_{s}} e_{i j}\left(u_{1}\right) e_{i j}(v) d x d t d y=-\iint_{Q \times Y_{s}} E_{i j}\left(\Psi_{0}\right) e_{i j}(v) d x d t d y
$$

for all $v \in L^{2}\left(Q ; \mathbb{H}_{\text {per }}^{1}\left(Y_{s} ; \mathbb{R}\right) / R_{\text {per }}^{s}\right)$. Hence, by extension, there exists a function $\Psi_{1} \in L^{2}\left(Q ; \mathbb{H}_{\text {per }}^{1}\left(Y_{s} ; \mathbb{R}\right)\right)$ such that

$$
\iint_{Q \times Y_{s}} e_{i j}\left(\Psi_{1}\right) e_{i j}(v) d x d t d y=-\iint_{Q \times Y_{s}} E_{i j}\left(\Psi_{0}\right) e_{i j}(v) d x d t d y
$$

for all $v \in L^{2}\left(Q ; \mathbb{H}_{p e r}^{1}(Y ; \mathbb{R})\right)$. 
Remark 2. We have

$\int_{Y_{s}} e_{i j}\left(\Psi_{1}(x, t)\right) d y=-\int_{Y_{s}} E_{i j}\left(\Psi_{0}(x, t)\right) d y$ a.e. in $Q$ for $i, j=1,2,3$.

Remark 3. Let $\mathcal{C}_{\text {per }}^{1}\left(Y_{s}\right)=\left\{v \in \mathcal{C}^{1}\left(\mathbb{R}^{3}\right): v, \frac{\partial v}{\partial y_{i}}(i=1,2,3)\right.$ are $Y$-periodic $\}$. Since $\mathcal{D}((0, T)) \otimes \mathcal{D}(\Omega) \otimes \mathcal{C}_{\text {per }}^{1}\left(Y_{s}\right)^{3}$ is dense in $L^{2}\left(Q ; H_{p e r}^{1}\left(Y_{s}\right)\right)$, we will choose in (3.22) test functions of the form $v=\Psi_{\varepsilon} \theta^{\varepsilon}$ with

$$
\Psi_{\varepsilon}=\Psi_{0}^{\varepsilon}+\varepsilon \Psi_{1 n}^{\varepsilon} \text { and } \theta=1 \text { in } \mathbb{R}
$$

$n \in \mathbb{N}$, where

$$
\left\{\begin{array}{l}
\Psi_{0} \in \mathcal{D}((0, T)) \otimes \mathcal{D}(\Omega) \otimes \mathcal{W} \\
\Psi_{1 n} \in \mathcal{D}((0, T)) \otimes \mathcal{D}(\Omega) \otimes \mathcal{C}_{p e r}^{1}(Y)^{3}, n \in \mathbb{N} \\
\text { the restriction }\left.\Psi_{1 n}\right|_{Q \times \widetilde{Y}_{s}} \text { of } \Psi_{1 n} \text { on } Q \times \widetilde{Y}_{s} \text { satisfying : } \\
\left.\Psi_{1 n}\right|_{Q \times \widetilde{Y}_{s}} \rightarrow \Psi_{1} \text { in } L^{2}\left(Q ; \mathbb{H}_{p e r}^{1}\left(Y_{s}\right)\right) \text { as } n \rightarrow \infty \\
\text { such that } \Psi_{1} \text { is the solution of }(3.30) \text { for } \Psi_{0} \text { above }
\end{array}\right.
$$

with

$\mathcal{W}=\left\{w \in \mathcal{C}_{\text {per }}^{1}(Y ; \mathbb{R})^{3}: \operatorname{div}_{y} w=0\right.$ in $Y_{f}$ and $e_{i j}(w)=0$ in $\left.Y_{s}(i, j=1,2,3)\right\}$,

and $\Psi_{0}^{\varepsilon}(x, t)=\Psi_{0}\left(x, t, \frac{x}{\varepsilon}\right), \quad \Psi_{1 n}^{\varepsilon}(x, t)=\Psi_{1 n}\left(x, t, \frac{x}{\varepsilon}\right) \quad(x, t) \in Q$.

3.3 Convergence theorem for the homogenization problem. According to Lemma 6, we need to define some vector spaces. It is wellknown that $E(\Omega)=\left\{u=\left(u^{i}\right) \in \mathbb{L}^{2}(\Omega): \operatorname{div} u \in L^{2}(\Omega)\right\}$ is a Hilbert space for the norm $\|u\|_{E(\Omega)}=\left(\|u\|_{\mathbb{L}^{2}(\Omega)}^{2}+\|\operatorname{div} u\|_{L^{2}(\Omega)}^{2}\right)^{\frac{1}{2}}, u \in E(\Omega)$, further $E_{0}(\Omega)=\left\{u \in E(\Omega ; \mathbb{R}): \gamma_{n} u=0\right\}$ is one of its closed vector subspace (cf. $[15])$, where $\gamma_{n}$ is the continuous linear operator from $E(\Omega)$ into $H^{-\frac{1}{2}}(\partial \Omega)$ such that $\gamma_{n} u(x)=u^{i}(x) n^{i}(x)$ for all $x \in \partial \Omega$ and all $u=\left(u^{i}\right) \in \mathcal{D}(\bar{\Omega})^{3}$, $n=\left(n^{i}\right)$ being the outer unit normal to $\partial \Omega$.

Now, the set

$W=\left\{w \in \mathbb{H}_{p e r}^{1}(Y ; \mathbb{R}): \operatorname{div}_{y} w=0\right.$ in $Y_{f}$ and $e_{i j}(w)=0$ in $\left.Y_{s}(i, j=1,2,3)\right\}$

is a closed vector subspace of $\mathbb{H}_{\text {per }}^{1}(Y ; \mathbb{R})$, and it is obvious that the mapping

$$
w \rightarrow\|w\|_{W}=\left(\|w\|_{\mathbb{L}^{2}(Y)}^{2}+\int_{Y_{f}} e_{i j}(w) e_{i j}(w) d y\right)^{\frac{1}{2}}
$$


from $W$ into $\mathbb{R}$ is a norm in $W$ equivalent to the usual norm of $\mathbb{H}_{p e r}^{1}(Y)$. Furthermore, the space $\mathcal{W}$ (of Remark 3) is dense in $W$. Let

$$
E_{0}(\Omega ; W)=\left\{u=\left(u^{i}\right) \in L^{2}(\Omega ; W): \widetilde{u} \in E_{0}(\Omega)\right\},
$$

where $\widetilde{u}=\left(\widetilde{u}^{i}\right), \quad \widetilde{u}^{i}$ being the function on $\Omega$ defined by $\widetilde{u}^{i}(x)=$ $\int_{Y} u^{i}(x, y) d y(x \in \Omega)$. Clearly, $E_{0}(\Omega ; W)$ is a vector subspace of $L^{2}(\Omega ; W)$. Furthermore, $E_{0}(\Omega ; W)$ is a Hilbert space for the norm

$$
\|u\|_{E_{0}(\Omega ; W)}=\left(\|u\|_{L^{2}(\Omega ; W)}^{2}+\|\operatorname{div} \widetilde{u}\|_{L^{2}(\Omega)}^{2}\right)^{\frac{1}{2}} \quad\left(u \in E_{0}(\Omega ; W)\right) .
$$

Theorem 2. Let $\left(u_{\varepsilon}\right)_{\varepsilon>0}$ be the sequence defined by Theorem 1. Then, as $\varepsilon \rightarrow 0$, we have

$$
u_{\varepsilon} \rightarrow \widetilde{u}_{0} \text { in } L^{\infty}\left(0, T ; E_{0}(\Omega)\right) \text {-weak } *,
$$

where $\widetilde{u}_{0}$ is the function from $Q$ into $\mathbb{R}^{3}$ defined by $\widetilde{u}_{0}^{i}(x, t)=$ $\int_{Y} u_{0}^{i}(x, t, y) d y \quad((x, t) \in Q), u_{0}=\left(u_{0}^{i}\right)$ being the unique function from $[0, T]$ into $L^{2}(\Omega ; W)$ satisfying

$$
\begin{gathered}
u_{0} \in L^{2}\left(0, T ; E_{0}(\Omega ; W)\right) \\
\frac{\partial u_{0}}{\partial t} \in L^{2}\left(0, T ; E_{0}(\Omega ; W)\right) \cap L^{\infty}\left(0, T ; L^{2}\left(\Omega ; \mathbb{H}_{p e r}^{1}(Y)\right)\right) \\
\frac{\partial^{2} u_{0}}{\partial t^{2}} \in L^{2}\left(0, T ; L^{2}\left(\Omega ; \mathbb{L}_{p e r}^{2}(Y)\right)\right)
\end{gathered}
$$

and the variational wave equation

$$
\begin{aligned}
& \iint_{\Omega \times Y} \rho \frac{\partial^{2} u_{0}^{i}}{\partial t^{2}}(t) v_{0}^{i} d x d y+\iint_{\Omega \times Y_{f}} \mu_{i j k h} e_{k h}\left(\frac{\partial u_{0}}{\partial t}(t)\right) e_{i j}\left(v_{0}\right) d x d y \\
& +\frac{\vartheta}{\left|Y_{f}\right|} \int_{\Omega} d i v \widetilde{u}_{0}(t) d i v \widetilde{v}_{0} d x=\int_{\Omega} f^{i}(t) \widetilde{v}_{0}^{i} d x \quad \text { for all } v_{0} \in E_{0}(\Omega ; W)
\end{aligned}
$$

$0<t<T$, with initial conditions

$$
u_{0}(0)=\frac{\partial u_{0}}{\partial t}(0)=\omega
$$

where $\mu_{i j k h}=\mu\left(\delta_{i k} \delta_{j h}+\delta_{i h} \delta_{j k}\right)$.

Proof. Step 1. Let us apply the weak two-scale convergence on a subsequence of $\left(z_{\varepsilon}\right)_{0<\varepsilon \leq \varepsilon_{0}}$. According to (3.23), we can extend each $z_{\varepsilon}$ 
such that

$$
z_{\varepsilon} \in L^{2}\left(0, T ; \widehat{\mathbb{H}_{0}^{1}(\Omega)}\right), 0<\varepsilon \leq \varepsilon_{0}, \text { and } \sup _{0<\varepsilon \leq \varepsilon_{0}}\left(\left\|z_{\varepsilon}\right\|_{L^{2}\left(0, T ; \mathbb{H}_{0}^{1}(\Omega)\right.}\right)<\infty
$$

Then, there exists a fundamental sequence extracted from $E^{\prime} \cap\left(0, \varepsilon_{0}\right]$, still denoted $E^{\prime}$ for simplicity, such that we have [10, Theorem 3], as $E^{\prime} \ni \varepsilon \rightarrow 0$,

$$
\int_{Q} E_{i j}\left(z_{\varepsilon}\right) \Phi^{\varepsilon} d x d t \rightarrow \iint_{Q \times Z}\left(E_{i j}(r)+e_{i j}\left(r_{1}\right)\right) \Phi d x d t d y d \tau
$$

for all $\Phi \in L^{2}\left(Q ; \mathcal{C}_{\text {per }}(Z)\right)$ and for $i, j=1,2,3$, where $r \in$ $L^{2}\left(0, T ; L_{\text {per }}^{2}\left(\Theta ; \mathbb{H}^{1}(\Omega)\right)\right), r_{1} \in L^{2}\left(Q ; L_{\text {per }}^{2}\left(\Theta ; \mathbb{H}_{\#}^{1}(Y)\right)\right)$.

Step 2. Here, we will derive (3.33) for a subsequence, further (3.34), (3.35), (3.36), (3.38). Thanks to Lemmas 5-9, it follows that $u_{\varepsilon} \in$ $L^{\infty}\left(0, T ; E_{0}(\Omega)\right)$ with $(3.33)$ as $E^{\prime} \ni \varepsilon \rightarrow 0$, and properties (3.34), (3.35), (3.36), (3.38). Now, we want to obtain (3.37).

Step 3. We will pass to the limit in (3.22) for a suitable test function. We take in (3.22) test functions of the form $v=\left(\Psi_{0}^{\varepsilon}+\varepsilon \Psi_{1 n}^{\varepsilon}\right) \theta^{\varepsilon}, n \in \mathbb{N}$, where $\Psi_{0}$ and $\Psi_{1 n}$ satisfy $(3.32), \theta \in \mathcal{C}_{\text {per }}(\Theta), \theta=1$. We obtain

$$
\begin{aligned}
& \int_{Q} \rho^{\varepsilon} \frac{\partial^{2} u_{\varepsilon}}{\partial t^{2}} \cdot \Psi_{0}^{\varepsilon} \theta^{\varepsilon} d x d t+\int_{Q_{f}^{\varepsilon}} \varepsilon b_{i j k h} E_{k h}\left(\frac{\partial u_{\varepsilon}}{\partial t}\right)\left(e_{i j}\left(\Psi_{0}\right)\right)^{\varepsilon} \theta^{\varepsilon} d x d t \\
& -\int_{Q_{f}^{\varepsilon}} p_{\varepsilon}\left(\operatorname{div} \Psi_{0}\right)^{\varepsilon} \theta^{\varepsilon} d x d t-\int_{Q_{f}^{\varepsilon}} p_{\varepsilon}\left(\operatorname{div}_{y} \Psi_{1 n}\right)^{\varepsilon} \theta^{\varepsilon} d x d t-\int_{Q} f \cdot \Psi_{0}^{\varepsilon} \theta^{\varepsilon} d x d t \\
& -\int_{Q} E_{i j}\left(z_{\varepsilon}\right) \chi_{s}^{\varepsilon}\left(E_{i j}\left(\Psi_{0}\right)\right)^{\varepsilon} \theta^{\varepsilon} d x d t-\int_{Q} E_{i j}\left(z_{\varepsilon}\right) \chi_{s}^{\varepsilon}\left(e_{i j}\left(\Psi_{1 n}\right)\right)^{\varepsilon} \theta^{\varepsilon} d x d t \\
& =\varepsilon \int_{Q} f \cdot \Psi_{1 n}^{\varepsilon} \theta^{\varepsilon} d x d t+\varepsilon \int_{Q_{f}^{\varepsilon}} \varepsilon b_{i j k h} E_{k h}\left(\frac{\partial u_{\varepsilon}}{\partial t}\right)\left(E_{i j}\left(\Psi_{0}\right)\right)^{\varepsilon} \theta^{\varepsilon} d x d t \\
& +\varepsilon \int_{Q_{f}^{\varepsilon}} p_{\varepsilon}\left(\operatorname{div} \Psi_{1 n}\right)^{\varepsilon} \theta^{\varepsilon} d x d t+\varepsilon \int_{Q_{s}^{\varepsilon}} E_{i j}\left(z_{\varepsilon}\right)\left(E_{i j}\left(\Psi_{1 n}\right)\right)^{\varepsilon} \theta^{\varepsilon} d x d t \\
& -\varepsilon \int_{Q} \rho^{\varepsilon} \frac{\partial^{2} u_{\varepsilon}}{\partial t^{2}} \cdot \Psi_{1 n}^{\varepsilon} \theta^{\varepsilon} d x d t+\varepsilon \int_{Q_{f}^{\varepsilon}} \varepsilon b_{i j k h} E_{k h}\left(\frac{\partial u_{\varepsilon}}{\partial t}\right)\left(e_{i j}\left(\Psi_{1 n}\right)\right)^{\varepsilon} \theta^{\varepsilon} d x d t \\
& -\varepsilon^{2} \int_{Q_{f}^{\varepsilon}} \varepsilon b_{i j k h} E_{k h}\left(\frac{\partial u_{\varepsilon}}{\partial t}\right)\left(E_{i j}\left(\Psi_{1 n}\right)\right)^{\varepsilon} \theta^{\varepsilon} d x d t+\frac{1}{\varepsilon} \int_{Q_{f}^{\varepsilon}} p_{\varepsilon}\left(\operatorname{div}_{y} \Psi_{0}\right)^{\varepsilon} \theta^{\varepsilon} d x d t \\
& +\frac{1}{\varepsilon} \int_{Q_{s}^{\varepsilon}} E_{i j}\left(z_{\varepsilon}\right)\left(e_{i j}\left(\Psi_{0}\right)\right)^{\varepsilon} \theta^{\varepsilon} d x d t
\end{aligned}
$$

where $\chi_{s}$ is the characteristic function of $\widetilde{Y}_{s}$ defined on $\mathbb{R}_{y}^{3}$. But now, since $\operatorname{div}_{y} \Psi_{0}=0$ in $Q \times Y_{f}$ and $e_{i j}\left(\Psi_{0}\right)=0$ in $Q \times Y_{s}(i, j=1,2,3)$, the 
last two terms at the right hand side are equal to zero. Next, we observe that $\chi_{s} E_{i j}\left(\Psi_{0}\right) \theta$ and $\chi_{s} e_{i j}\left(\Psi_{1 n}\right) \theta$ belong to $\mathcal{C}\left(\bar{Q} ; L_{p e r}^{\infty}(Z)\right)$. Thus, by using (3.39), Lemmas 6 and 9, at the limit (when $E^{\prime} \ni \varepsilon \rightarrow 0$ ), we conclude that the right-hand side converges to zero, and the left-hand side converges to

$$
\begin{aligned}
& \iint_{Q \times Y} \rho \frac{\partial^{2} u_{0}^{i}}{\partial t^{2}} \Psi_{0}^{i} d x d t d y+\iint_{Q \times Y_{f}} \mu_{i j k h} e_{k h}\left(\frac{\partial u_{0}}{\partial t}\right) e_{i j}\left(\Psi_{0}\right) d x d t d y \\
& -\iint_{Q} p\left(\int_{Y_{f}} \operatorname{div}_{y} \Psi_{1 n} d y\right) d x d t-\iint_{Q \times Y_{f}} p \operatorname{div} \Psi_{0} d x d t d y \\
& -\iiint_{Q \times Y_{s} \times \Theta}\left(E_{i j}(r)+e_{i j}\left(r_{1}\right)\right)\left(E_{i j}\left(\Psi_{0}\right)+e_{i j}\left(\Psi_{1 n}\right)\right) d x d t d y d \tau \\
& -\iint_{Q \times Y} f^{i} \Psi_{0}^{i} d x d t d y \quad \text { for all } n \in \mathbb{N} .
\end{aligned}
$$

Letting $z=\int_{\Theta} r d \tau$ and $z_{1}=\int_{\Theta} r_{1} d \tau$, we have $z \in L^{2}\left(0, T ; \mathbb{H}^{1}(\Omega)\right), z_{1} \in$ $L^{2}\left(Q ; \mathbb{H}_{p e r}^{1}(Y)\right)$ with $E_{i j}(z)=\int_{\Theta} E_{i j}(r) d \tau$ and $e_{i j}\left(z_{1}\right)=\int_{\Theta} e_{i j}\left(r_{1}\right) d \tau$. Further it follows that for all $n \in \mathbb{N}$

$$
\begin{aligned}
& \iint_{Q \times Y} \rho \frac{\partial^{2} u_{0}^{i}}{\partial t^{2}} \Psi_{0}^{i} d x d t d y+\iint_{Q \times Y_{f}} \mu_{i j k h} e_{k h}\left(\frac{\partial u_{0}}{\partial t}\right) e_{i j}\left(\Psi_{0}\right) d x d t d y \\
& -\iint_{Q} p\left(\int_{Y_{f}} \operatorname{div}_{y} \Psi_{1 n} d y\right) d x d t-\iint_{Q \times Y_{f}} p \operatorname{div} \Psi_{0} d x d t d y \\
& =\iint_{Q \times Y} f^{i} \Psi_{0}^{i} d x d t d y \\
& \quad+\iint_{Q \times Y_{s}}\left(E_{i j}(z)+e_{i j}\left(z_{1}\right)\right)\left(E_{i j}\left(\Psi_{0}\right)+e_{i j}\left(\Psi_{1 n}\right)\right) d x d t d y .
\end{aligned}
$$

Step 4. Derivation of (3.37). Let us consider the function $\xi=\left(\xi^{i}\right)$ from $\widetilde{Y}_{s}$ into $\mathbb{R}^{3}$ defined by : for $k=\left(k_{i}\right) \in \mathbb{Z}^{3}$ and $y \in Y_{s}, \xi^{i}(y+k)=k_{i}$, $i=1,2,3$. Then the function $y \rightarrow y-\xi(y)$ from $\tilde{Y}_{s}$ into $\mathbb{R}^{3}$ belongs to $\mathbb{H}_{\text {per }}^{1}\left(Y_{s}\right)$.

Since $-\int_{Y_{f}} \operatorname{div}_{y} \Psi_{1 n} d y=\int_{Y_{s}} \operatorname{div}_{y} \Psi_{1 n} d y$ in $Q$, we have

$$
\begin{aligned}
-\iint_{Q \times Y_{f}} p \operatorname{div}_{y} \Psi_{1 n} d x d t d y & =\iint_{Q \times Y_{s}} p \operatorname{div}_{y} \Psi_{1 n} d x d t d y \\
& =\iint_{Q \times Y_{s}} e_{i j}\left(\Psi_{1 n}\right) e_{i j}(p(y-\xi(y))) d x d t d y
\end{aligned}
$$


According to (3.30) and (3.32) it follows that, as $n \rightarrow \infty$,

$$
\begin{aligned}
-\iint_{Q \times Y_{f}} p \operatorname{div}_{y} \Psi_{1 n} d x d t d y & \rightarrow-\iint_{Q \times Y_{s}} E_{i j}\left(\Psi_{0}\right) e_{i j}(p(y-\xi(y))) d x d t d y \\
& =-\iint_{Q \times Y_{s}} p \operatorname{div} \Psi_{0} d x d t d y
\end{aligned}
$$

On the other hand, according to the convergence in (3.32), it follows that, up to a subsequence, still denoted $\left(\Psi_{1 n}\right)_{n}$,

$$
\begin{aligned}
\left.\iint_{Q \times Y_{s}} E_{i j}(z)\right)\left(E_{i j}\left(\Psi_{0}\right)+e_{i j}\left(\Psi_{1 n}\right)\right) d x d t d y \rightarrow \\
\iint_{Q \times Y_{s}} E_{i j}(z)\left(E_{i j}\left(\Psi_{0}\right)+e_{i j}\left(\Psi_{1}\right)\right) d x d t d y
\end{aligned}
$$

as $n \rightarrow \infty$. Therefore

$$
\begin{aligned}
\iint_{Q \times Y_{s}}\left(E_{i j}(z)+e_{i j}\left(z_{1}\right)\right)\left(E_{i j}\left(\Psi_{0}\right)+e_{i j}\left(\Psi_{1 n}\right)\right) d x d t d y \rightarrow \\
\iint_{Q \times Y_{s}}\left(E_{i j}(z)+e_{i j}\left(z_{1}\right)\right)\left(E_{i j}\left(\Psi_{0}\right)+e_{i j}\left(\Psi_{1}\right)\right) d x d t d y .
\end{aligned}
$$

But we have

$$
\begin{aligned}
& \iint_{Q \times Y_{s}} E_{i j}(z)\left(E_{i j}\left(\Psi_{0}\right)+e_{i j}\left(\Psi_{1}\right)\right) d x d t d y \\
& =\int_{Q} E_{i j}(z(x, t))\left(\int_{Y_{s}}\left(E_{i j}\left(\Psi_{0}(x, t)\right)+e_{i j}\left(\Psi_{1}(x, t)\right)\right) d y\right) d x d t=0 \text { (Remark 2) }
\end{aligned}
$$

and

$$
\iint_{Q \times Y_{s}} e_{i j}\left(z_{1}\right)\left(E_{i j}\left(\Psi_{0}\right)+e_{i j}\left(\Psi_{1}\right)\right) d x d t d y=0 \text { (Lemma 12). }
$$

Consequently

$$
\begin{aligned}
& \iint_{Q \times Y} \rho \frac{\partial^{2} u_{0}^{i}}{\partial t^{2}} \Psi_{0}^{i} d x d t d y+\iint_{Q \times Y_{f}} \mu_{i j k h} e_{k h}\left(\frac{\partial u_{0}}{\partial t}\right) e_{i j}\left(\Psi_{0}\right) d x d t d y \\
& -\iint_{Q \times Y} \frac{\partial p}{\partial x_{i}} \Psi_{0}^{i} d x d t d y=\iint_{Q \times Y} f^{i} \Psi_{0}^{i} d x d t d y .
\end{aligned}
$$


Clearly (3.16) implies

$$
\begin{aligned}
& \iint_{Q \times Y} \rho \frac{\partial^{2} u_{0}^{i}}{\partial t^{2}} \Psi_{0}^{i} d x d t d y+\iint_{Q \times Y_{f}} \mu_{i j k h} e_{k h}\left(\frac{\partial u_{0}}{\partial t}\right) e_{i j}\left(\Psi_{0}\right) d x d t d y \\
& +\frac{\vartheta}{\left|Y_{f}\right|} \int_{Q} \operatorname{div} \widetilde{u}_{0} \operatorname{div}\left(\int_{Y} \Psi_{0} d y\right) d x d t=\int_{Q} f^{i}\left(\int_{Y} \Psi_{0}^{i} d y\right) d x d t
\end{aligned}
$$

for all $\Psi_{0} \in \mathcal{D}(\Omega) \otimes \mathcal{W}$. This yields (3.37) thanks to the density of $\mathcal{D}(\Omega) \otimes \mathcal{W}$ into $E_{0}(\Omega ; W)$, where

$$
\widetilde{v}_{0}(x, t)=\int_{Y} v_{0}(x, t, y) d y=\left(\int_{Y} v_{0}^{i}(x, t, y) d y\right) .
$$

Step 5. It remains to prove that $u_{0}$ is the unique function satisfying (3.33)-(3.38). Let us assume that $f=\omega$ and $v_{0}=\frac{\partial u_{0}}{\partial t}(t)$ in (3.37). Then, we have

$$
\begin{aligned}
& \frac{d}{d s}\left(\iint_{\Omega \times Y} \rho \frac{\partial u_{0}^{i}}{\partial t}(s) \frac{\partial u_{0}^{i}}{\partial t}(s) d x d y+\frac{\vartheta}{\left|Y_{f}\right|} \int_{\Omega} \operatorname{div} \widetilde{u}_{0}(s) \operatorname{div} \widetilde{u}_{0}(s) d x\right) \\
& +2 \iint_{\Omega \times Y_{f}} \mu_{i j k h} e_{k h}\left(\frac{\partial u_{0}}{\partial t}(s)\right) e_{i j}\left(\frac{\partial u_{0}}{\partial t}(s)\right) d x d y=0 \quad(0<s<t<T) .
\end{aligned}
$$

Integrating from 0 to $t$, and using the initial conditions (see Lemma 7), we obtain

$$
\begin{aligned}
& \iint_{\Omega \times Y} \rho \frac{\partial u_{0}^{i}}{\partial t}(t) \frac{\partial u_{0}^{i}}{\partial t}(t) d x d y+\int_{\Omega} \operatorname{div} \widetilde{u}_{0}(t) \operatorname{div} \widetilde{u}_{0}(t) d x \\
& +\int_{0}^{t} \iint_{\Omega \times Y_{f}} \mu_{i j k h} e_{k h}\left(\frac{\partial u_{0}}{\partial t}(s)\right) e_{i j}\left(\frac{\partial u_{0}}{\partial t}(s)\right) d x d y d s \leq 0,0<t<T .
\end{aligned}
$$

Therefore $\left\|u_{0}\right\|_{L^{\infty}\left(0, T ; E_{0}(\Omega ; W)\right)}=0$, and $u_{0}$ of Lemma 6 is the unique function from $[0, T]$ into $E_{0}(\Omega ; W)$ satisfying (3.33)-(3.38).

3.4 Physical description in brief of the local problem. Let $u_{0}$ be the function defined in Theorem 2. There exists a negligible set $\mathcal{N}_{0} \subset \Omega$ such that for $x \in \Omega \backslash \mathcal{N}_{0}$ the function $t \rightarrow u_{0}(x, t, \cdot)$ from $[0, T]$ into $\mathbb{L}_{\text {per }}^{2}(Y)$, denoted by $u_{0}(x)$, belongs to $L^{2}\left(0, T ; \mathbb{L}_{p e r}^{2}(Y)\right)$. Thanks to the isometric isomorphism between the spaces $L^{2}\left(0, T ; L^{2}\left(\Omega ; \mathbb{L}_{p e r}^{2}(Y)\right)\right)$ and $L^{2}\left(\Omega ; L^{2}\left(0, T ; \mathbb{L}_{p e r}^{2}(Y)\right)\right)$, we show easily that $u_{0}(x), \quad \frac{\partial}{\partial t} u_{0}(x) \in$ $L^{2}\left(0, T ; \mathbb{H}_{\text {per }}^{1}(Y)\right)$ and $\frac{\partial^{2}}{\partial t^{2}} u_{0}(x) \in L^{2}\left(0, T ; \mathbb{L}_{\text {per }}^{2}(Y)\right)$ with $\frac{\partial}{\partial t} u_{0}(x)=$ $\frac{\partial u_{0}}{\partial t}(x, \cdot, \cdot)$ and $\frac{\partial^{2}}{\partial t^{2}} u_{0}(x)=\frac{\partial^{2} u_{0}}{\partial t^{2}}(x, \cdot, \cdot)$ for all $x \in \Omega \backslash \mathcal{N}(\mathcal{N}$ being a negligible set such that $\mathcal{N}_{0} \subset \mathcal{N} \subset \Omega$ ). 
Theorem 3. Let $u_{0}$ be the function in Theorem 2. For almost all $x \in \Omega, u_{0}(x)$ is uniquely determined by $u_{0}(x) \in \mathcal{C}([0, T] ; W), \frac{\partial}{\partial t} u_{0}(x) \in$ $L^{2}(0, T ; W) \cap \mathcal{C}\left([0, T] ; \mathbb{L}_{p e r}^{2}(Y)\right), \quad \frac{\partial^{2}}{\partial t^{2}} u_{0}(x) \in L^{2}\left(0, T ; \mathbb{L}_{\text {per }}^{2}(Y)\right)$ and the variational problem

$$
\begin{aligned}
& \int_{Y} \rho \frac{\partial^{2}}{\partial t^{2}} u_{0}^{i}(x)(t) w^{i} d y+\int_{Y_{f}} \mu_{i j k h} e_{k h}\left(\frac{\partial}{\partial t} u_{0}(x)(t)\right) e_{i j}(w) d y \\
& =\left(f^{i}(x, t)-\frac{\partial p}{\partial x_{i}}(x, t)\right) \int_{Y} w^{i} d y, \quad w \in W, \quad 0<t<T,
\end{aligned}
$$

with initial conditions

$$
u_{0}(x)(0)=\frac{\partial}{\partial t} u_{0}(x)(0)=\omega .
$$

Proof. It suffices to take in (3.37) test functions of the form $v_{0}=\varphi \otimes w$, $\varphi \in \mathcal{D}(\Omega), w \in W$, to obtain (3.40). Let us prove (3.41) : Let $v \in \mathcal{C}_{T}^{1}\left([0, T] ; \mathbb{L}_{p e r}^{2}(Y)\right)=\left\{v \in \mathcal{C}^{1}\left([0, T] ; \mathbb{L}_{p e r}^{2}(Y)\right): v(T)=0\right\}$. We have

$$
\iint_{] 0, T[\times Y} u_{0}(x) v^{\prime} d t d y=-\int_{Y} u_{0}(x)(0) v(0) d y-\iint_{] 0, T[\times Y} \frac{\partial}{\partial t} u_{0}(x) v d t d y .
$$

We multiply both the members by $\varphi(x), x \in \Omega \backslash \mathcal{N}, \varphi \in \mathcal{D}(\Omega)$, and we integrate on $\Omega \backslash \mathcal{N}$. It results that

$$
\begin{aligned}
\iint_{Q \times Y} u_{0} \varphi & \frac{\partial v}{\partial t} d x d t d y= \\
& -\iint_{\Omega \times Y} u_{0}(x)(0) \varphi(x) v(0) d x d y-\iint_{Q \times Y} \frac{\partial u_{0}}{\partial t} \varphi v d x d t d y
\end{aligned}
$$

for all $\varphi \in \mathcal{D}(\Omega)$ and all $v \in \mathcal{C}_{T}^{1}\left([0, T] ; \mathbb{L}_{p e r}^{2}(Y)\right)$. Since $\frac{\partial u_{0}}{\partial t}(0)=\omega$ (Lemma $7)$, it follows that $\int_{Y} u_{0}(x)(0) w d y=0$ for all $w \in \mathbb{L}_{p e r}^{2}(Y)$. Therefore $u_{0}(x)(0)=\omega$ for all $x \in \Omega \backslash \mathcal{N}$. We proceed as above, where $u_{0}(x)$ is replaced by $\frac{\partial}{\partial t} u_{0}(x)$, to obtain $\frac{\partial}{\partial t} u_{0}(x)(0)=\omega$.

We use the same arguments as in theorem 1 to end the proof.

The local problem (3.40)-(3.41) describes the motion of an incompressible viscous fluid with solid rigid particles in suspension. It results to (3.40) that

$$
\begin{aligned}
& \int_{Y_{f}} \rho_{f} \frac{\partial^{2}}{\partial t^{2}} u_{0}^{i}(x)(t) w^{i} d y+\int_{Y_{f}} \mu_{i j k h} e_{k h}\left(\frac{\partial}{\partial t} u_{0}(x)(t)\right) e_{i j}(w) d y \\
& -\left(f^{i}(x, t)-\frac{\partial p}{\partial x_{i}}(x, t)\right) \int_{Y_{f}} w^{i} d y=0
\end{aligned}
$$


for all $w \in \mathbb{H}_{\text {per }}^{1}\left(Y_{f}\right), w=\omega$ on $\widetilde{\partial Y}_{s}$ and $\operatorname{div}_{y} w=0$. Then, for almost all $(x, t) \in Q$ there exists $p_{1}(x, t) \in L_{\text {per }}^{2}\left(Y_{f}\right) / \mathbb{R}=$ $\left\{v \in L_{p e r}^{2}\left(Y_{f}\right): \int_{Y_{f}} v d y=0\right\}$ such that (see, e.g., [9])

$$
\begin{aligned}
& \rho_{f} \frac{\partial^{2}}{\partial t^{2}} u_{0}^{i}(x)(t)-\frac{\partial}{\partial y_{j}}\left(\mu_{i j k h} e_{k h}\left(\frac{\partial}{\partial t} u_{0}(x)(t)\right)\right)+\frac{\partial p}{\partial x_{i}}(x, t) \\
& +\frac{p_{1}(x, t)}{\partial y_{i}}=f^{i}(x, t) \quad \text { in } Y_{f} \quad(i=1,2,3) .
\end{aligned}
$$

But the distribution $\rho_{f} \frac{\partial^{2}}{\partial t^{2}} u_{0}^{i}(x)-\frac{\partial}{\partial y_{j}}\left(\mu_{i j k h} e_{k h}\left(\frac{\partial}{\partial t} u_{0}(x)\right)\right)+\frac{\partial p}{\partial x_{i}}(x)-f^{i}(x)$ belongs to $L^{2}\left(0, T ; H_{\text {per }}^{-1}\left(Y_{f}\right)\right)\left[H_{\text {per }}^{-1}\left(Y_{f}\right)\right.$ denoting the dual of the space $\left\{w \in H_{\text {per }}^{1}\left(Y_{f}\right): w=0\right.$ on $\left.\left.\widetilde{\partial Y}_{s}\right\}\right]$; so that $\frac{p_{1}(x, t)}{\partial y_{i}}=\frac{p_{1}(x)}{\partial y_{i}}(t)$. On letting

$$
\sigma_{i j}^{0}(x)=-\delta_{i j} p_{1}(x)+\mu_{i j k h} e_{k h}\left(\frac{\partial}{\partial t} u_{0}(x)\right), \quad i=1,2,3,
$$

we have $\frac{\partial \sigma_{i j}^{0}(x)}{\partial y_{j}} \in L^{2}\left(0, T ; L_{p e r}^{2}\left(Y_{f}\right)\right), \quad i, j=1,2,3$, and the partial differential equations which govern this motion are (for almost all $x \in \Omega$ ) :

$$
\begin{aligned}
& \rho_{f} \frac{\partial^{2}}{\partial t^{2}} u_{0}(x)=\operatorname{div}_{y} \sigma^{0}(x)+f(x)-D p(x) \text { in } Y_{f} \times(0, T) \\
& \operatorname{div}_{y} u_{0}(x)=0 \text { in } Y_{f} \times(0, T) \\
& e_{i j}\left(u_{0}(x)\right)=0 \text { in } Y_{s} \times(0, T) \quad(i, j=1,2,3) \\
& u_{0}(x) \text { is } Y \text {-periodic }
\end{aligned}
$$

where $D p=\left(\frac{\partial p}{\partial x_{i}}\right)_{i=1,2,3}$ and $\sigma^{0}(x)=\left(\sigma_{i j}^{0}(x)\right)_{i, j=1,2,3}$. Otherwise, since (3.40) is equivalent to

$$
\begin{aligned}
& \int_{Y_{f}} \rho_{f} \frac{\partial^{2}}{\partial t^{2}} u_{0}^{i}(x) w^{i} d y+\int_{Y_{f}} \mu_{i j k h} e_{k h}\left(\frac{\partial}{\partial t} u_{0}(x)\right) e_{i j}(w) d y \\
& -\left(f^{i}(x)-\frac{\partial p}{\partial x_{i}}(x)\right) \int_{Y_{f}} w^{i} d y=-\int_{Y_{s}}\left(\rho_{s} \frac{\partial^{2}}{\partial t^{2}} u_{0}^{i}(x)-f^{i}(x)+\frac{\partial p}{\partial x_{i}}(x)\right) w^{i} d y
\end{aligned}
$$

for all $w \in W$, we add $-\int_{Y_{f}} p_{1}(x) \operatorname{div}_{y} w d y$ to both sides and, by using Green formula combining with the first equation in (3.42), we obtain

$-\int_{Y_{s}}\left(\rho_{s} \frac{\partial^{2}}{\partial t^{2}} u_{0}^{i}(x)-f^{i}(x)+\frac{\partial p}{\partial x_{i}}(x)\right) w^{i} d y+\left[\sigma_{i j}^{0}(x) n^{j}, \gamma_{\circ}^{s} w^{i}\right]_{s}=0$ for all $w \in W$

where $\gamma_{\circ}^{s}$ is the trace operator on $\partial Y_{s}, n=\left(n^{i}\right)$ is the outer unit normal to $\partial Y_{s}$ with respect to $Y_{s},[,]_{s}$ denotes the scalar product in the duality between $H^{-\frac{1}{2}}\left(\partial Y_{s}\right)$ and $H^{\frac{1}{2}}\left(\partial Y_{s}\right)$. Since $w(y)=a+b \wedge y$ in $Y_{s}\left(a, b \in \mathbb{R}^{3}\right)$, 
it follows that with $\mathcal{I}=(1,1,1)$

$$
\begin{aligned}
& \int_{Y_{s}}\left(\rho_{s} \frac{\partial^{2}}{\partial t^{2}} u_{0}^{i}(x)-f^{i}(x)+\frac{\partial p}{\partial x_{i}}(x)\right) d y+\left[\sigma_{i j}^{0}(x) n^{j}, 1\right]_{s}=0 \\
& \int_{Y_{s}} y \wedge\left(\rho_{s} \frac{\partial^{2}}{\partial t^{2}} u_{0}(x)-f(x)+D p(x)\right) d y+\left[y \wedge \sigma^{0}(x) n, \mathcal{I}\right]_{s}=0 .
\end{aligned}
$$

Remark 4. If $\sigma_{i j}^{0}(x) n^{j} \in L^{2}\left(\partial Y_{s}\right), \quad i=1,2,3$, then we have $\left[\sigma_{i j}^{0}(x) n^{j}, 1\right]_{s}=\int_{\partial Y_{s}} \sigma_{i j}^{0}(x) n^{j} d \sigma$ and $\left[y \wedge \sigma^{0}(x) n, \mathcal{I}\right]_{s}=\int_{\partial Y_{s}} y \wedge\left(\sigma^{0}(x) n\right) d \sigma$, where $d \sigma$ is the superficial measure on $\partial Y_{s}$.

\section{The homogenization result in the case} $f \in L^{2}\left(0, T ; \mathbb{L}^{2}(\Omega)\right)$

4.1 Preliminaries. In the sequel, we assume that $f$ is fixed in $L^{2}\left(0, T ; \mathbb{L}^{2}(\Omega)\right)$. Then, it results just both the first and second estimates of Lemma 5 . Therefore we have the following lemma, where we recall that $\left(u_{\varepsilon}\right)_{\varepsilon>0}$ is the sequence obtained in Theorem 1. In what follows, $E$ designates a fundamental sequence.

Lemma 13. A subsequence $E^{\prime}$ can be extracted from $E$ such that, as $E^{\prime} \ni \varepsilon \rightarrow 0$, we have

$$
\begin{aligned}
& u_{\varepsilon}^{i} \rightarrow u_{0}^{i} \text { in } L^{2}(Q) \text {-weak two-scale, } i=1,2,3, \\
& u_{\varepsilon} \rightarrow \widetilde{u}_{0} \text { in } L^{\infty}\left(0, T ; E_{0}(\Omega)\right) \text {-weak } *,
\end{aligned}
$$

where $\widetilde{u}_{0}=\left(\widetilde{u}_{0}^{i}\right)=\left(\int_{Y} u_{0}^{i}(\cdot, \cdot, y) d y\right), u_{0}=\left(u_{0}^{i}\right)$ being a function from $[0, T]$ into $W$ satisfying

$$
u_{0} \in L^{2}\left(0, T ; E_{0}(\Omega ; W)\right), \frac{\partial u_{0}}{\partial t} \in L^{2}\left(0, T ; L^{2}(\Omega ; W)\right), \text { and } u_{0}(0)=\omega .
$$

Proof. As in the proof of Lemmas 6 and 7, all those properties follow to first and second estimates of Lemma 5 .

Now, let $\left(f_{n}\right)_{n \in \mathbb{N}^{*}}$ be a sequence in $\mathcal{C}^{1}\left([0, T] ; \mathbb{L}^{2}(\Omega)\right)$ such that $f_{n}(0)=\omega$ for all $n \in \mathbb{N}^{*}$ and

$$
\left\|f_{n}-f\right\|_{L^{2}\left(0, T ; \mathbb{L}^{2}(\Omega)\right)} \rightarrow 0 \text { as } n \rightarrow \infty .
$$

According to Theorem 1, there exists exactly one sequence $\left(u_{n \varepsilon}\right)_{n \in \mathbb{N}^{*}, \varepsilon>0}$ such that, given $n \in \mathbb{N}^{*}$ and $\varepsilon>0, u_{n \varepsilon}$ is the unique function from $[0, T]$ into $V_{\varepsilon}$ defined by : 


$$
\begin{aligned}
& u_{n \varepsilon} \in \mathcal{C}\left([0, T] ; V_{\varepsilon}\right), \\
& \frac{\partial u_{n \varepsilon}}{\partial t} \in \mathcal{C}\left([0, T] ; \mathbb{L}^{2}(\Omega)\right) \cap L^{2}\left(0, T ; V_{\varepsilon}\right), \frac{\partial^{2} u_{n \varepsilon}}{\partial t^{2}} \in L^{2}\left(0, T ; \mathbb{L}^{2}(\Omega)\right)
\end{aligned}
$$

and

$$
\begin{gathered}
\int_{\Omega} \rho^{\varepsilon} \frac{\partial^{2} u_{n \varepsilon}}{\partial t^{2}}(t) \cdot v d x+\varepsilon^{2} b^{\varepsilon}\left(\frac{\partial u_{n \varepsilon}}{\partial t}(t), v\right)+a^{\varepsilon}\left(u_{n \varepsilon}(t), v\right) \\
=\int_{\Omega} f_{n}(t) \cdot v d x \quad \text { for all } v \in V_{\varepsilon}(0<t<T), \\
u_{n \varepsilon}(0)=\frac{\partial u_{n \varepsilon}}{\partial t}(0)=\omega .
\end{gathered}
$$

Lemma 14. Let $u_{0}$ and $E^{\prime}$ be defined in Lemma 13. Then we have

$$
\begin{aligned}
\lim _{E^{\prime} \ni \varepsilon \rightarrow 0}\left(\lim _{n \rightarrow \infty} \int_{Q} u_{n \varepsilon}^{i} \Psi^{\varepsilon} d x d t\right) & =\iint_{Q \times Y} u_{0}^{i} \Psi d x d t d y \\
& =\lim _{n \rightarrow \infty}\left(\lim _{E^{\prime} \ni \varepsilon \rightarrow 0} \int_{Q} u_{n \varepsilon}^{i} \Psi^{\varepsilon} d x d t\right)
\end{aligned}
$$

for all $\Psi \in L^{2}\left(Q ; \mathcal{C}_{p e r}(Y)\right), i=1,2,3$.

Proof. Step 1. Let us prove the first equality. Let $\varepsilon \in E^{\prime}$ be fixed. We show easily that $\left(u_{n \varepsilon}\right)_{n \in \mathbb{N}^{*}}$ is a Cauchy sequence in $L^{2}\left(0, T ; \mathbb{L}^{2}(\Omega)\right)$, and

$$
\lim _{n \rightarrow \infty} \int_{Q} u_{n \varepsilon}^{i} \Psi^{\varepsilon} d x d t=\int_{Q} u_{\varepsilon}^{i} \Psi^{\varepsilon} d x d t \text { for all } \Psi \in L^{2}\left(Q ; \mathcal{C}_{p e r}(Y)\right), i=1,2,3,
$$

where $u_{\varepsilon}=\left(u_{\varepsilon}^{i}\right)$ is a term of the sequence $\left(u_{\varepsilon}\right)_{\varepsilon \in E^{\prime}}$ of Lemma 13. The first equality of Lemma 14 result to (4.1).

Step 2. Now, let us prove the second equality. It follows by Theorem 2 that, for each $n \in \mathbb{N}^{*}$,

$$
\lim _{E^{\prime} \ni \varepsilon \rightarrow 0} \int_{Q} u_{n \varepsilon}^{i} \Psi^{\varepsilon} d x d t=\iint_{Q \times Y} u_{n 0}^{i} \Psi d x d t d y
$$

for all $\Psi \in L^{2}\left(Q ; \mathcal{C}_{p e r}(Y)\right), \quad i=1,2,3$, where $u_{n 0}=\left(u_{n 0}^{i}\right)$ is the unique function from $[0, T]$ into $W$ satisfying

$$
\begin{aligned}
& u_{n 0} \in L^{2}\left(0, T ; E_{0}(\Omega ; W)\right), \\
& \frac{\partial u_{n 0}}{\partial t} \in L^{2}\left(0, T ; E_{0}(\Omega ; W)\right) \cap L^{\infty}\left(0, T ; L^{2}\left(\Omega ; \mathbb{H}_{p e r}^{1}(Y)\right)\right)
\end{aligned}
$$


and

$$
\frac{\partial^{2} u_{n 0}}{\partial t^{2}} \in L^{2}\left(Q ; \mathbb{L}_{p e r}^{2}(Y)\right)
$$

and the variational problem

$$
\begin{array}{r}
\iint_{\Omega \times Y} \rho \frac{\partial^{2} u_{n 0}^{i}}{\partial t^{2}}(t) v_{0}^{i} d x d y+\iint_{\Omega \times Y_{f}} \mu_{i j k h} e_{k h}\left(\frac{\partial u_{n 0}}{\partial t}(t)\right) e_{i j}\left(v_{0}\right) d x d y \\
(4.6)+\frac{\vartheta}{\left|Y_{f}\right|} \int_{\Omega} \operatorname{div} \widetilde{u}_{n 0}(t) \operatorname{div} \widetilde{v}_{0} d x=\int_{\Omega} f_{n}^{i}(t) \widetilde{v}_{0}^{i} d x \quad \text { for all } v_{0} \in E_{0}(\Omega ; W),
\end{array}
$$

$0<t<T$, with initial conditions

$$
u_{n 0}(0)=\frac{\partial u_{n 0}}{\partial t}(0)=\omega
$$

Since $n$ is arbitrarily fixed, we obtain the a priori estimate

$$
\begin{aligned}
& \left\|\frac{\partial u_{n 0}}{\partial t}-\frac{\partial u_{m 0}}{\partial t}\right\|_{L^{\infty}\left(0, T ; L^{2}\left(\Omega ; \mathbb{L}^{2}(Y)\right)\right)}^{2}+\sum_{i, j=1}^{3}\left\|e_{i j}\left(\frac{\partial u_{n 0}}{\partial t}-\frac{\partial u_{m 0}}{\partial t}\right)\right\|_{L^{2}\left(Q \times Y_{f}\right)}^{2} \\
& (4.7)+\left\|\operatorname{div}\left(\widetilde{u}_{n 0}-\widetilde{u}_{m 0}\right)\right\|_{L^{\infty}\left(0, T ; L^{2}(\Omega)\right)}^{2} \leq c\left\|f_{n}-f_{m}\right\|_{L^{2}\left(0, T ; \mathbb{L}^{2}(\Omega)\right)}^{2},
\end{aligned}
$$

for all $m, n \in \mathbb{N}^{*}, m \neq n$, where the constant $c$ does not depend on $m$ and $n$. Hence $\left(u_{n 0}\right)_{n \in \mathbb{N}^{*}}$ is a Cauchy sequence in $L^{2}\left(Q ; \mathbb{L}_{p e r}^{2}(Y)\right)$. There exists $u_{\infty} \in L^{2}\left(Q ; \mathbb{L}_{p e r}^{2}(Y)\right)$ such that $u_{n 0} \rightarrow u_{\infty}$ in $L^{2}\left(Q ; \mathbb{L}_{p e r}^{2}(Y)\right)$ as $n \rightarrow \infty$. This strong limit implies

$$
\lim _{n \rightarrow \infty} \iint_{Q \times Y} u_{n 0}^{i} \Psi d x d t d y=\iint_{Q \times Y} u_{\infty}^{i} \Psi d x d t d y\left(\Psi \in L^{2}\left(Q ; \mathcal{C}_{p e r}(Y)\right)\right),
$$

for $i=1,2,3$. Combining with (4.5), it follows that

$\lim _{n \rightarrow \infty}\left(\lim _{E^{\prime} \ni \varepsilon \rightarrow 0} \int_{Q} u_{n \varepsilon}^{i} \Psi^{\varepsilon} d x d t\right)=\iint_{Q \times Y} u_{\infty}^{i} \Psi d x d t d y\left(\Psi \in L^{2}\left(Q ; \mathcal{C}_{p e r}(Y)\right)\right)$,

for $i=1,2,3$.

Let us prove that $u_{0}=u_{\infty}$ in $L^{2}\left(Q ; \mathbb{L}^{2}(Y)\right)$. We fix $\delta>0$. Let $\Psi \in L^{2}\left(Q ; \mathcal{C}_{\text {per }}(Y)\right)$ be arbitrary fixed, further put

$$
\begin{aligned}
& a_{n \varepsilon}^{i}=\iint_{Q} u_{n \varepsilon}^{i} \Psi^{\varepsilon} d x d t, a_{\varepsilon}^{i}=\int_{Q} u_{\varepsilon}^{i} \Psi^{\varepsilon} d x d t, a_{n}^{i}=\iint_{Q \times Y} u_{n 0}^{i} \Psi d x d t d y \\
& a_{\infty}^{i}=\iint_{Q \times Y} u_{\infty}^{i} \Psi d x d t d y, \text { and } a_{0}^{i}=\iint_{Q \times Y} u_{0}^{i} \Psi d x d t d y .
\end{aligned}
$$


There are $n_{1} \in \mathbb{N}^{*}$ and $\varepsilon_{1} \in E^{\prime}$ such that

$$
\left|a_{n_{1}}^{i}-a_{\infty}^{i}\right|<\frac{\delta}{4},\left|a_{n_{1} \varepsilon_{1}}^{i}-a_{\varepsilon_{1}}^{i}\right|<\frac{\delta}{4},\left|a_{\varepsilon_{1}}^{i}-a_{0}^{i}\right|<\frac{\delta}{4} \text {, and }\left|a_{n_{1} \varepsilon_{1}}^{i}-a_{n_{1}}^{i}\right|<\frac{\delta}{4}
$$

for $i=1,2,3$. Therefore

$$
\left|a_{\infty}^{i}-a_{0}^{i}\right| \leq\left|a_{\infty}^{i}-a_{n_{1}}^{i}\right|+\left|a_{n_{1}}^{i}-a_{n_{1} \varepsilon_{1}}^{i}\right|+\left|a_{n_{1} \varepsilon_{1}}^{i}-a_{\varepsilon_{1}}^{i}\right|+\left|a_{\varepsilon_{1}}^{i}-a_{0}^{i}\right|<\delta .
$$

Since $\delta>0$ is arbitrarily fixed, we obtain the desired equality.

Thanks to the density of $L^{2}\left(Q ; \mathcal{C}_{\text {per }}(Y)\right)$ in $L^{2}\left(Q ; L_{\text {per }}^{2}(Y)\right)$, we deduce that $\left\|u_{\infty}^{i}-u_{0}^{i}\right\|_{L^{2}(Q \times Y)}=0$ for each $i=1,2,3$.

Remark 5. It results to (4.7) that $\left(\frac{\partial u_{n 0}}{\partial t}\right)_{n \in \mathbb{N}^{*}}$ is a Cauchy sequence in $L^{2}(Q ; W)$, so that there is a function $v_{\infty} \in L^{2}(Q ; W)$ such that

$$
\frac{\partial u_{n 0}}{\partial t} \rightarrow v_{\infty} \text { in } L^{2}(Q ; W) \text { as } n \rightarrow \infty .
$$

According to the continuity of the derivation operator in in the distributional space $\mathcal{D}^{\prime}\left((0, T) ; L^{2}(\Omega ; W)\right)$, one proves that $v_{\infty}=\frac{\partial u_{\infty}}{\partial t}=$ $\frac{\partial u_{0}}{\partial t}$.

4.2 Convergence theorem for the homogenization problem. We are now capable to prove the following

Theorem 4. Let $\left(u_{\varepsilon}\right)_{\varepsilon>0}$ be the sequence defined by Theorem 1. When $\varepsilon \rightarrow 0$,

$$
u_{\varepsilon} \rightarrow \widetilde{u}_{0} \quad \text { in } L^{\infty}\left(0, T ; E_{0}(\Omega)\right) \text {-weak } *
$$

where $u_{0}$ is the unique function from $[0, T]$ into $L^{2}(\Omega ; W)$ satisfying

$$
\begin{gathered}
u_{0} \in L^{\infty}\left(0, T ; E_{0}(\Omega ; W)\right), \\
\frac{\partial u_{0}}{\partial t} \in L^{2}\left(0, T ; L^{2}(\Omega ; W)\right),
\end{gathered}
$$

and the variational equation

$$
\begin{aligned}
& \frac{d}{d t} \iint_{\Omega \times Y} \rho \frac{\partial u_{0}}{\partial t}(t) \cdot v_{0} d x d y+\iint_{\Omega \times Y_{f}} \mu_{i j k h} e_{k h}\left(\frac{\partial u_{0}}{\partial t}(t)\right) e_{i j}\left(v_{0}\right) d x d y \\
& \quad+\frac{\vartheta}{\left|Y_{f}\right|} \int_{\Omega} \operatorname{div\widetilde {u}_{0}}(t) d i v \widetilde{v}_{0} d x=\int_{\Omega} f(t) \cdot \widetilde{v}_{0} d x
\end{aligned}
$$


for all $v_{0} \in E_{0}(\Omega ; W), 0<t<T$ combined with initial conditions

$$
u_{0}(0)=\frac{\partial u_{0}}{\partial t}(0)=\omega
$$

Proof. By virtue of Lemma 13, it suffices to prove that the weak twoscale limit (4.1) satisfies (4.11)-(4.12), further it is the unique solution of this variational problem. We will proceed in three steps.

Step 1. Let us derive (4.11). We multiply (4.6) by $\phi(t), 0<t<T$ fixed, $\phi \in \mathcal{D}((0, T))$ and integrate by parts from 0 to $T$ to obtain

$$
\begin{aligned}
& -\iint_{Q \times Y} \rho \frac{\partial u_{n 0}^{i}}{\partial t}(t) v_{0}^{i} \phi^{\prime}(t) d x d y d t \\
& +\iint_{Q \times Y_{f}} \mu_{i j k h} e_{k h}\left(\frac{\partial u_{n 0}}{\partial t}(t)\right) e_{i j}\left(v_{0} \phi(t)\right) d x d y d t \\
& +\frac{\vartheta}{\left|Y_{f}\right|} \int_{Q} \operatorname{div} \widetilde{u}_{n 0}(t) \operatorname{div} \widetilde{v}_{0} \phi(t) d x d t=\int_{Q} f_{n}^{i}(t) \widetilde{v}_{0}^{i} \phi(t) d x d t \quad\left(v_{0} \in E_{0}(\Omega ; W)\right) .
\end{aligned}
$$

We pass to the limit, as $n \rightarrow \infty$, and it follows by (4.3) combined with Lemma 14 and Remark 5 that

$$
\begin{aligned}
& -\iint_{Q \times Y} \rho \frac{\partial u_{0}^{i}}{\partial t}(t) v_{0}^{i} \phi^{\prime}(t) d x d y d t \\
& +\iint_{Q \times Y_{f}} \mu_{i j k h} e_{k h}\left(\frac{\partial u_{0}}{\partial t}(t)\right) e_{i j}\left(v_{0} \phi(t)\right) d x d y d t \\
& +\frac{\vartheta}{\left|Y_{f}\right|} \int_{Q} \operatorname{div} \widetilde{u}_{0}(t) \operatorname{div} \widetilde{v}_{0} \phi(t) d x d t=\int_{Q} f^{i}(t) \widetilde{v}_{0}^{i} \phi(t) d x d t
\end{aligned}
$$

This leads to the variational equation (4.11).

Step 2. In (4.11)-(4.12), it remains to prove that $\frac{\partial u_{0}}{\partial t}(0)=\omega$. Let $H(\Omega ; W)$ be the closure of $L^{2}(\Omega ; W)$ in $L^{2}\left(\Omega ; \mathbb{L}_{p e r}^{2}(Y)\right)$. We endow $H(\Omega ; W)$ with the hilbertian norm

$$
\|u\|_{H(\Omega ; W)}=\left(\iint_{\Omega \times Y} \rho u^{i} u^{i} d x d y\right)^{\frac{1}{2}} \quad\left(u=\left(u^{i}\right) \in H(\Omega ; W)\right) .
$$

The associated scalar product will be still denoted $[\cdot, \cdot]$. We will assume that $H(\Omega ; W)$ is identified to its dual. Since $\mathcal{D}(\Omega) \otimes W$ is dense in $L^{2}(\Omega ; W)$, one has the following continuous canonical injections (with density)

$$
E_{0}(\Omega ; W) \hookrightarrow L^{2}(\Omega ; W) \hookrightarrow H(\Omega ; W) \hookrightarrow L^{2}\left(\Omega ; W^{\prime}\right) \hookrightarrow E_{0}(\Omega ; W)^{\prime},
$$


where $W^{\prime}$ and $E_{0}(\Omega ; W)^{\prime}$ are the duals of $W$ and $E_{0}(\Omega ; W)$ respectively. Then, we will also denote by $[\cdot, \cdot]$ the duality pairing between $E_{0}(\Omega ; W)^{\prime}$ and $E_{0}(\Omega ; W)$. Let $A$ be a continuous linear operator from $E_{0}(\Omega ; W)$ into $E_{0}(\Omega ; W)^{\prime}$ and $B$ a continuous linear operator from $L^{2}(\Omega ; W)$ into $E_{0}(\Omega ; W)^{\prime}$ defined by

$$
\begin{aligned}
& {[A v, w]=\frac{\vartheta}{\left|Y_{f}\right|} \int_{\Omega} \operatorname{div} \widetilde{v} \operatorname{div} \widetilde{w} d x \quad\left(v, w \in E_{0}(\Omega ; W)\right),} \\
& {[B v, w]=\iint_{\Omega \times Y_{f}} \mu_{i j k h} e_{k h}(v) e_{i j}(w) d x d y \quad\left(v \in L^{2}(\Omega ; W), w \in E_{0}(\Omega ; W)\right) .}
\end{aligned}
$$

Let $L$ be the unique function of $L^{2}(0, T ; H(\Omega ; W))$ such that for almost all $t \in[0, T]$,

$$
\int_{\Omega} f^{i}(t) \widetilde{v}^{i} d x=[L(t), v] \text { for all } v \in H(\Omega ; W) .
$$

Then (4.11) becomes

$$
\frac{d}{d t}\left[\frac{\partial u_{0}}{\partial t}(t), v_{0}\right]=\left[L(t)-B \frac{\partial u_{0}}{\partial t}(t)-A u_{0}(t), v_{0}\right]
$$

for all $v_{0} \in E_{0}(\Omega ; W), 0<t<T$. Since $\frac{\partial u_{0}}{\partial t}$ and $L-B \frac{\partial u_{0}}{\partial t}-$ $A u_{0}$ are elements of $L^{2}\left(0, T ; E_{0}(\Omega ; W)^{\prime}\right)$, then $\frac{d}{d t}\left(\frac{\partial u_{0}}{\partial t}\right)$ belongs to $L^{2}\left(0, T ; E_{0}(\Omega ; W)^{\prime}\right)$ with $\frac{d}{d t}\left(\frac{\partial u_{0}}{\partial t}\right)+B \frac{\partial u_{0}}{\partial t}+A u_{0}=L$. Therefore we have $\frac{\partial u_{0}}{\partial t} \in \mathcal{C}\left([0, T] ; E_{0}(\Omega ; W)^{\prime}\right)$, so that $\frac{\partial u_{0}}{\partial t}(0)$ is well defined. Multiplying (4.11) by $\phi(t), t \in(0, T), \phi \in \mathcal{C}_{T}^{1}([0, T])$, next integrating by parts from 0 to $T$ yields

$$
\begin{aligned}
-\left[\frac{\partial u_{0}}{\partial t}(0), v_{0}\right] \phi(0)-\iint_{Q \times Y} \rho \frac{\partial u_{0}^{i}}{\partial t}(t) v_{0}^{i} \phi^{\prime}(t) d x d y d t \\
+\iint_{Q \times Y_{f}} \mu_{i j k h} e_{k h}\left(\frac{\partial u_{0}}{\partial t}(t)\right) e_{i j}\left(v_{0} \phi(t)\right) d x d y d t \\
+\frac{\vartheta}{\left|Y_{f}\right|} \int_{Q} \operatorname{div} \widetilde{u}_{0}(t) \operatorname{div} \widetilde{v}_{0} \phi(t) d x d t \\
=\int_{Q} f^{i}(t) \widetilde{v}_{0}^{i} \phi(t) d x d t .
\end{aligned}
$$

On the other hand, multiplying (4.6) by $\phi(t), t \in(0, T), \phi \in \mathcal{C}_{T}^{1}([0, T])$, then integration by parts from 0 to $T$ yields 


$$
\begin{aligned}
& -\iint_{Q \times Y} \rho \frac{\partial u_{n 0}^{i}}{\partial t}(t) v_{0}^{i} \phi^{\prime}(t) d x d y d t \\
& \quad+\iint_{Q \times Y_{f}} \mu_{i j k h} e_{k h}\left(\frac{\partial u_{n 0}}{\partial t}(t)\right) e_{i j}\left(v_{0} \phi(t)\right) d x d y d t \\
& \quad \frac{\vartheta}{\left|Y_{f}\right|} \int_{Q} \operatorname{div} \widetilde{u}_{n 0}(t) \operatorname{div} \widetilde{v}_{0} \phi(t) d x d t=\int_{Q} f_{n}^{i}(t) \widetilde{v}_{0}^{i} \phi(t) d x d t, \quad n \in \mathbb{N}^{*}
\end{aligned}
$$

(condition $\frac{\partial u_{n 0}}{\partial t}(0)=\omega$ being taking into account). When $n \rightarrow \infty$, one obtains equation (4.13). Combining with (4.16), we are drived to

$$
\left[\frac{\partial u_{0}}{\partial t}(0), v_{0}\right]=0 \text { for all } v_{0} \in E_{0}(\Omega ; W)
$$

that is $\frac{\partial u_{0}}{\partial t}(0)=\omega\left(\right.$ in $\left.E_{0}(\Omega ; W)^{\prime}\right)$.

Step 3. Let us derive (4.8). The uniqueness of the solution $u_{0}$ in the problem (4.11)-(4.12) is proved in the above section, by assuming that $f=\omega$ (then $f \in \mathcal{C}^{1}\left([0, T] ; \mathbb{L}^{2}(\Omega)\right)$ with $\left.f(0)=\omega\right)$. Consequently, we can replace the fundamental sequence $E^{\prime}$ in Lemma 13 by all the sequence $0<\varepsilon \leq 1$. The proof follows.

\section{References}

[1] G. Allaire, Homogenization and two-scale convergence, SIAM J. Math. Anal., 23 (1992), 1482-1518.

[2] A.C. Biazutti, On a nonlinear evolution equation and its applications, Nonlinear Analysis TMA, 24 (1995), 1221-1234.

[3] N. Bourbaki, Intégration, Hermann, Paris, 1966, chapters 1-4.

[4] N. Bourbaki, Intégration, Hermann, Paris, 1966, chapter 5.

[5] H. Brezis, Analyse fonctionnelle : Théorie et applications, Masson, Paris 1983.

[6] I. Hlavacek and J. Necas, On inequalities of Korn's type II : Application to linear Elasticity, Arch. Rational Mech. Anal., 36 (1970), 312-334.

[7] D. Lukkassen, G. Nguetseng and P. Wall, Two-scale convergence, Int. J. Pure and Appl. Math., 1 (2002), 35-86.

[8] S. Migórski, Homogenization of hyperbolic-parabolic equations in perforated domains, Acta Math., (1996), 59-72. 
[9] G. Nguetseng, Un problème raide intervenant en mécanique des mélanges, Bull. Sc. Math., 107 (1983), 393-411.

[10] G. Nguetseng, A general convergence result for a functional related to the theory of homogenization, SIAM J. Math. Anal., 20 (1989), 608623.

[11] G. Nguetseng, Asymptotic analysis for a stiff variational problem arising in mechanics, SIAM J. Math. Anal., 21 (1990), 1394-1414.

[12] G. Nguetseng, Homogenization structures and Applications I, Z. Anal. Andwend., 22 (2003), 203-221.

[13] H. Nnang, Un théorème d'existence et d'unicité, Preprint, University of Yaounde I, 2000.

[14] E. Sanchez-Palencia, Nonhomogeneous media and vibration theory, Lect. Notes in Physics 127, Springer-verlag, Berlin, New-York, 1980.

[15] R. Temam, Navier-Stokes equations, North-Holland, 1979.

University of Yaounde I

Faculty of Sciences, Department of Mathematics

P.O. Box 812 Yaounde

Cameroon

(E-mail : nguetsengg@yahoo.fr)

University of Yaounde I

Ecole Normale Supérieure

Department of Mathematics

P.O. Box 47 Yaounde

Cameroon

(E-mail : hnnang@yahoo.fr)

Gothenburg University

Department of Mathematical Sciences

SE-412 96 Gothenburg

Sweden

(E-mail : nilss@math.chalmers.se)

(Received : December 2007) 


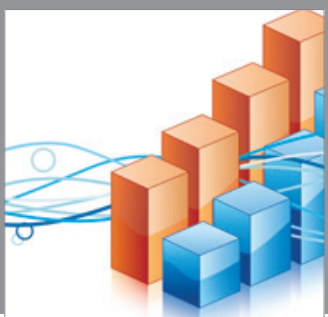

Advances in

Operations Research

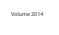

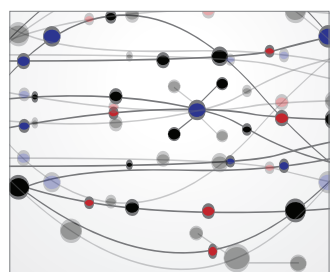

\section{The Scientific} World Journal
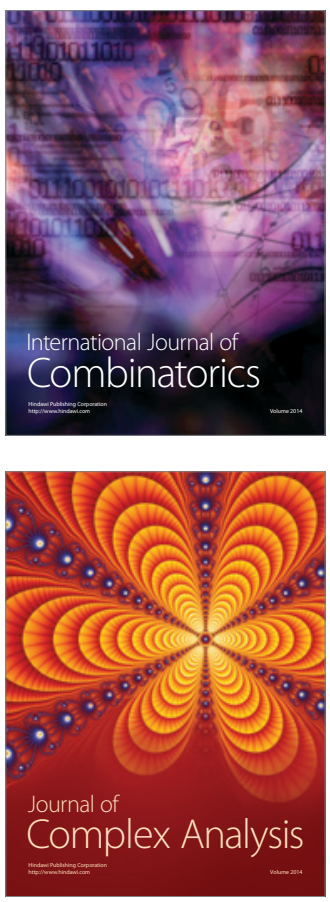

International Journal of

Mathematics and

Mathematical

Sciences
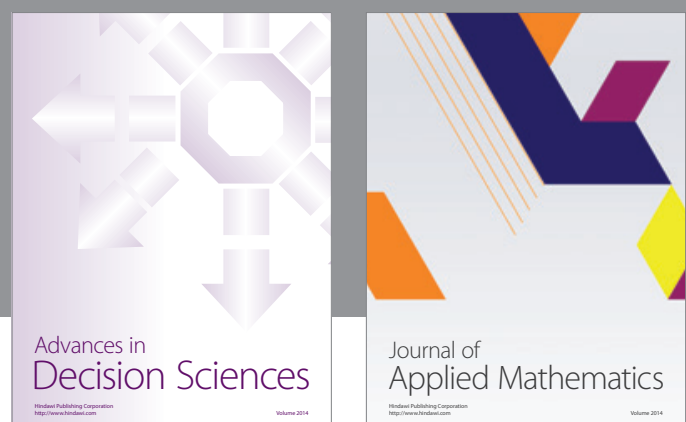

Journal of

Applied Mathematics
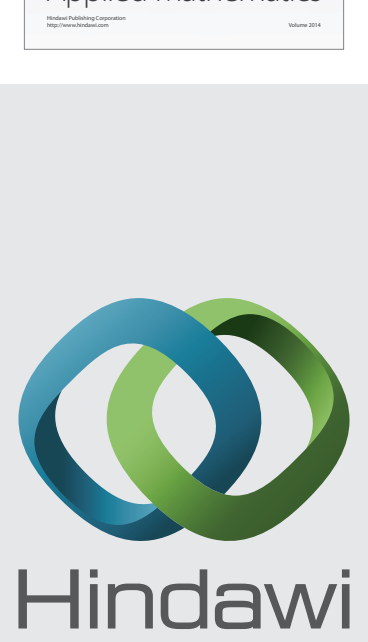

Submit your manuscripts at http://www.hindawi.com
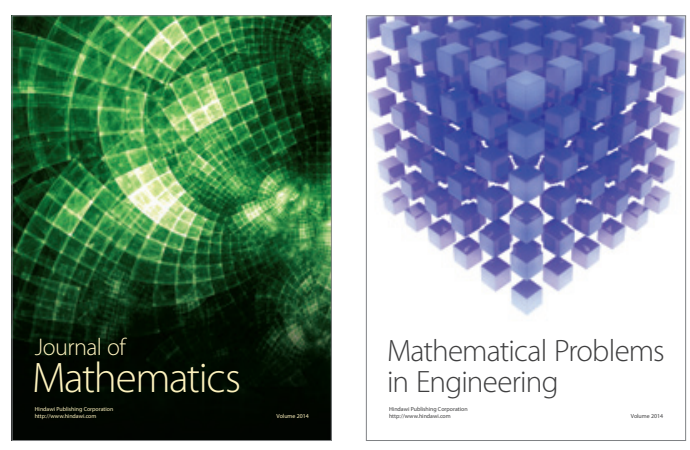

Mathematical Problems in Engineering
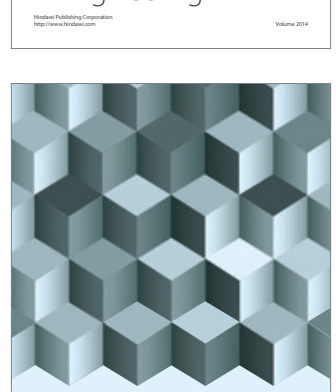

Journal of

Function Spaces
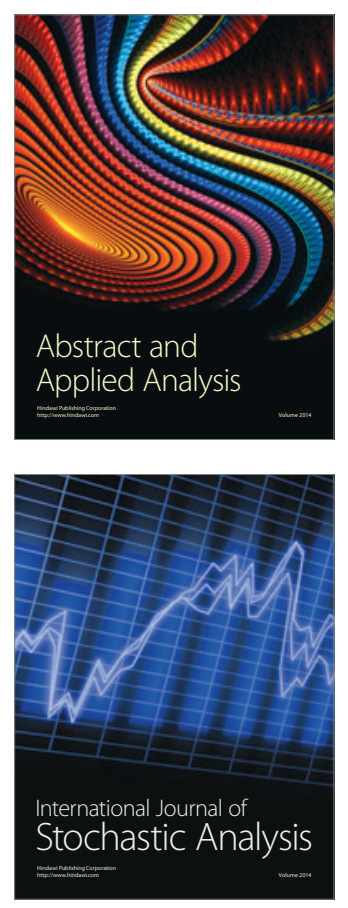

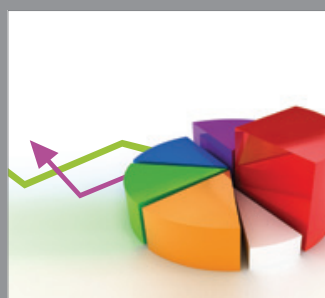

ournal of

Probability and Statistics

Promensencen
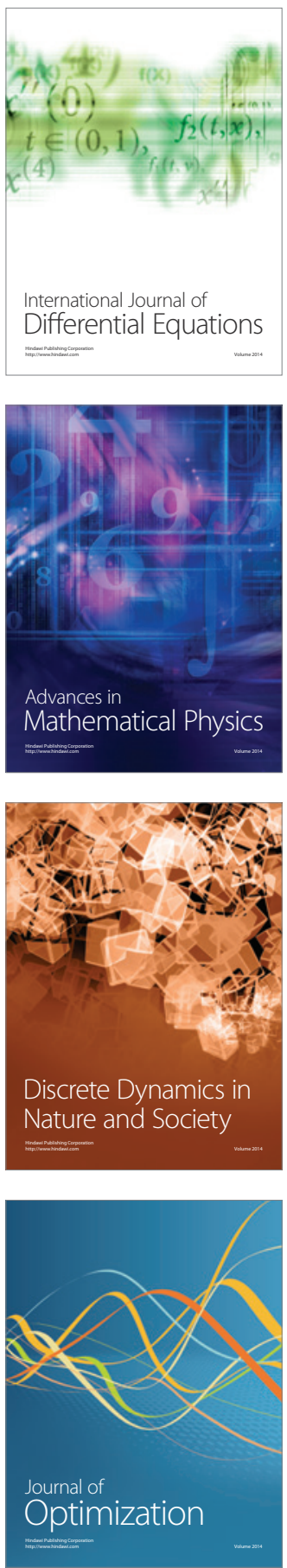